\title{
ON THE LOCAL REGULARITY \\ OF SOLUTIONS IN LINEAR VISCOELASTICITY OF SEVERAL SPACE DIMENSIONS
}

\author{
JONG UHN KIM
}

\begin{abstract}
In this paper we discuss the local regularity of solutions of a nonlocal system of equations which describe the motion of a viscoelastic medium in several space dimensions. Our main tool is the microlocal analysis combined with MacCamy's trick and the argument of the classical energy method.
\end{abstract}

\section{INTRODUCTION}

In this paper we discuss the local regularity of solutions of an integro-differential system which describes the motion of a linear viscoelastic medium in several space dimensions. The system is given by the following equations:

$$
\begin{aligned}
u_{t t}^{i}(t, x)= & C_{i j}^{\alpha \beta}(t, x) \frac{\partial^{2}}{\partial x_{\alpha} \partial x_{\beta}} u^{j}(t, x)+D_{i j}^{\alpha}(t, x) \frac{\partial}{\partial x_{\alpha}} u^{j}(t, x) \\
& +\int_{0}^{t} M_{i j}^{\alpha \beta}(t, s, x) \frac{\partial^{2}}{\partial x_{\alpha} \partial x_{\beta}} u^{j}(s, x) d s \\
& +\int_{0}^{t} N_{i j}^{\alpha}(t, s, x) \frac{\partial}{\partial x_{\alpha}} u^{j}(s, x) d s, \quad \text { for } i=1, \ldots, n,
\end{aligned}
$$

in $(0, \infty) \times R^{n}$,

$$
u(0, x)=u_{0}(x), \quad u_{t}(0, x)=u_{1}(x), \quad \text { in } R^{n},
$$

where $x=\left(x_{1}, \ldots, x_{n}\right) \in R^{n}$ and $u=\left(u^{1}, \ldots, u^{n}\right)$ denotes the displacement from equilibrium. We employ the convention of summation on repeated indices. The system $(0.1)$ stands for the conservation of linear momentum. The integral terms are accountable for the effect of memory. Since these terms are nonlocal, the problem addressed in the present paper is a new kind in the subject of local regularity of solutions. by

The constituent law for a linear viscoelastic material with memory is given

$$
\sigma_{i \alpha}(t, x)=\int_{-\infty}^{t} K_{i j}^{\alpha \beta}(t-s, x) \varepsilon_{j \beta}(s, x) d s,
$$

Received by the editors August 21, 1992.

1991 Mathematics Subject Classification. Primary 35B65, 35L99, 73F15.

Key words and phrases. Local regularity, MacCamy's trick, propagation of singularities, energy method, microlocal regularity, bicharacteristic strip, bicharacteristic curve, singular support. 
where $\sigma_{i \alpha}$ is the stress tensor and $\varepsilon_{j \beta}$ is the strain tensor defined by

$$
\varepsilon_{j \beta}=\frac{1}{2}\left(\frac{\partial u^{j}}{\partial x_{\beta}}+\frac{\partial u^{\beta}}{\partial x_{j}}\right) .
$$

The kernel function $K_{i j}^{\alpha \beta}$ is given by

$$
K_{i j}^{\alpha \beta}(t, x)=G_{i j}^{\alpha \beta}(0, x) \delta(t)+H(t) \frac{\partial}{\partial t} G_{i j}^{\alpha \beta}(t, x),
$$

where $G_{i j}^{\alpha \beta}(t, x)$ is the relaxation function and $\delta(t), H(t)$ are the delta and Heaviside step functions, respectively. Since the relaxation function is derived from the density function of the stored energy, it satisfies the symmetry condition:

$$
G_{i j}^{\alpha \beta}=G_{\alpha j}^{i \beta}=G_{j i}^{\beta \alpha} .
$$

The conservation of linear momentum is expressed by

$$
\rho \frac{\partial^{2}}{\partial t^{2}} u^{i}=\frac{\partial}{\partial x_{\alpha}} \sigma_{i \alpha}+f_{i}, \quad \text { for } i=1, \ldots, n,
$$

where $\rho=\rho(t, x)$ is the density of the material and $f=\left(f_{1}, \ldots, f_{n}\right)$ stands for the external force. We assume that the medium was undisturbed before the initial time $t=0$, and that there is no external force. Then, $(0.1)$ follows from (0.3) through (0.7). We note that if the density $\rho$ depends on time, then the convolution structure of the memory kernel is broken. When the past history of the material for $t<0$ is taken into account, it can be easily incorporated into the external force. Since the system is linear, we may use the superposition so that the effect of the external force can be separately studied by the same method of analysis with zero initial conditions. In fact, if the external force is $C^{\infty}$-smooth, then we may simply ignore it in view of our purpose.

The system $(0.1)$ is by now well understood in the context of the Cauchy problem and the initial-boundary value problem; see [3]. There are also some results on the nonlinear system. For extensive references, see [14]. On the other hand, the investigation of wave propagation and the effect of a memory term on the regularity of solutions is not complete yet, even though there are many earlier works on this. We cite [2], [5], [7], [10], [12], [13], and [14] among others.

Here we focus on the local regularity of solutions in conjunction with the propagation of singularities. Since the memory term is nonlocal, we cannot directly apply the known results on the propagation of singularities in linear elasticity. In fact, the nature of singularities in linear viscoelasticity is different from that in linear elasticity. The main difference lies in the possible emergence of stationary singularities. These singularities are weaker than those carried by the initial data, and they do not move away. This interesting phenomenon was first pointed out in [5], and was completely analyzed in [10] for a linear homogeneous viscoelastic medium in one space dimension. The authors of [10] used the Laplace transform and obtained precise results on the propagation of singularities for various kinds of memory kernels. In particular, they showed that if the memory kernel is smooth, stationary singularities can appear. In the framework of local regularity, this result was extended to a one-dimensional 
nonhomogeneous medium in my previous work [11]. The method of [11] is different from those of all earlier works. It consists of the following three tools:

(i) MacCamy's trick,

(ii) Hörmander's result on the propagation of singularities, and

(iii) the argument of the classical energy method.

In the present paper, we still employ these tools to work out a similar result in several space dimensions. However, completely new technical difficulties arise in the case of several space dimensions, particularly in using MacCamy's trick. The purpose of MacCamy's trick is to control the integral term, which is nonlocal. It consists in rewriting the original equation in an equivalent form where the integral term is of lower order. Obviously, it is easier to handle the integral term if the order becomes lower. For implementation of MacCamy's trick, we should solve a Volterra integral equation. In one space dimension, this is a fairly simple matter. But in several space dimensions, the integral kernel is a matrix pseudodifferential operator of order zero. This is a new kind of problem, and we present all the details for this in $\S 3$.

We shall outline the contents of other sections. In $\S 1$, we state the main result and sketch a general strategy of the proof. In order to justify the statement of the main result, we need a general existence theorem and the domain of dependence of solutions which is technically necessary in the proof of the main result. Hence, in $\S 2$, we establish the existence, uniqueness, and domain of dependence of solutions to the Cauchy problem. The existence of solutions is known in some particular function spaces. But we need it in a more general function space. The domain of dependence seems to be also known, at least as folklore. However, we could not find any appropriate reference which provides the proof of these results according to our need. Therefore, we present the details of the proof rather than beg the readers to believe the theorem. After all these preparations, we give the details of the proof of the main result in $\S 4$.

This paper has not discussed reflection of singularities on the boundary. It has been investigated by many people for hyperbolic equations, and it is known that the propagation of singularities can be very complex depending on the geometry of the boundary. In linear elasticity, it is also known that there can be singularities that run along the boundary. They are called Rayleigh waves, and were investigated in [16]. In case of a nonlocal system of equations, propagation of singularities near the boundary seems to be wide open for future investigation.

Finally, I would like to express my gratitude to Professor M. Renardy for useful information on this subject. I am also grateful to the anonymous referee for constructive comments.

\section{Notation.}

$$
\begin{gathered}
\mathscr{C}^{n}=\text { the set of all } n \text {-tuples of complex numbers, } \\
Z_{+}=\text {the set of all nonnegative integers, } \\
Z_{+}^{n}=\text { the set of all } n \text {-tuples of nonnegative integers. }
\end{gathered}
$$

We shall employ the following multi-index notation. For $\alpha=\left(\alpha_{1}, \ldots, \alpha_{n}\right) \in$ $Z_{+}^{n}$,

$$
|\alpha|=\alpha_{1}+\cdots+\alpha_{n}, \quad \alpha !=\alpha_{1} ! \cdots \alpha_{n} !
$$

For $\alpha, \beta \in Z_{+}^{n}, \beta \leq \alpha$ means $\beta_{i} \leq \alpha_{i}$, for each $i$. If $\alpha, \beta \in Z_{+}^{n}$ and $\beta \leq \alpha$, 
we write

$$
\left(\begin{array}{l}
\alpha \\
\beta
\end{array}\right)=\left(\begin{array}{l}
\alpha_{1} \\
\beta_{1}
\end{array}\right) \cdots\left(\begin{array}{c}
\alpha_{n} \\
\beta_{n}
\end{array}\right)=\frac{\alpha !}{\beta !(\alpha-\beta) !} .
$$

For $\alpha \in Z_{+}^{n}, x \in R^{n}, \xi \in R^{n}$, and $t \in R$,

$$
\begin{gathered}
\partial_{x}^{\alpha}=\left(\frac{\partial}{\partial x_{1}}\right)^{\alpha_{1}} \cdots\left(\frac{\partial}{\partial x_{n}}\right)^{\alpha_{n}}, \quad \partial_{\xi}^{\alpha}=\left(\frac{\partial}{\partial \xi_{1}}\right)^{\alpha_{1}} \cdots\left(\frac{\partial}{\partial \xi_{n}}\right)^{\alpha_{n}}, \\
D_{j}=-\sqrt{-1} \frac{\partial}{\partial x_{j}}, \quad \text { for } j=1, \ldots, n, \\
D_{x}^{\alpha}=D_{1}^{\alpha_{1}} \cdots D_{n}^{\alpha_{n}}, \quad D_{t}=-\sqrt{-1} \frac{\partial}{\partial t} .
\end{gathered}
$$

For $m \in R, H^{m}\left(R^{n}\right)$ denotes the usual Sobolev space equipped with the norm

$$
\|f\|_{H^{m}\left(R^{n}\right)}=\left\|(1+|\xi|)^{m} \hat{f}(\xi)\right\|_{L^{2}\left(R^{n}\right)},
$$

where $\hat{f}(\xi)$ is the Fourier transform of $f(x)$. When $f$ is $\mathscr{C}^{N}$ valued and each component belongs to $H^{m}\left(R^{n}\right)$, we still write $f \in H^{m}\left(R^{n}\right)$. We also follow this convention for other function classes.

The following notation is standard. For an open subset $\Omega$ of $R^{n}$,

$\mathscr{D}^{\prime}(\Omega)=$ the space of distributions over $\Omega$,

$$
W F(u)=\text { the wave front set of } u \text {, for } u \in \mathscr{D}^{\prime}(\Omega) \text {. }
$$

For $u \in \mathscr{D}^{\prime}(\Omega)$, we say that $u$ is $H^{m}$ at $x_{0} \in \Omega$ if there is a function $\phi(x) \epsilon$ $C_{0}^{\infty}(\Omega)$ such that $\phi(x)=1$ in a neighborhood of $x_{0}$ and $\phi u \in H^{m}\left(R^{n}\right)$.

For $m \in R$ and $T>0$, we write

$$
\begin{aligned}
\mathscr{S}^{m} & \left([0, T] \times[0, T] \times R^{n} \times R^{n}\right) \\
& =\text { the set of all complex valued } a(t, s, x, \xi) \\
& \in C^{\infty}\left([0, T] \times[0, T] \times R^{n} \times R^{n}\right) \\
& \text { which satisfies the following condition: }
\end{aligned}
$$

for each $(\alpha, \beta, \gamma, \delta) \in Z_{+} \times Z_{+} \times Z_{+}^{n} \times Z_{+}^{n}$, there is a positive constant $K_{\alpha \beta \gamma \delta}$ such that

$$
\left|\partial_{t}^{\alpha} \partial_{s}^{\beta} \partial_{x}^{\gamma} \partial_{\xi}^{\delta} a(t, s, x, \xi)\right| \leq K_{\alpha \beta \gamma \delta}(1+|\xi|)^{m-|\delta|},
$$

for all $(t, s, x, \xi) \in[0, T] \times[0, T] \times R^{n} \times R^{n}$.

We also use some subsets of $\mathscr{S}^{m}\left([0, T] \times[0, T] \times R^{n} \times R^{n}\right)$ :

$$
\mathscr{S}^{m}\left([0, T] \times R^{n} \times R^{n}\right)=\text { the set of all elements }
$$
of $\mathscr{S}^{m}\left([0, T] \times[0, T] \times R^{n} \times R^{n}\right)$ which are independent of $s$,

$$
\begin{aligned}
\mathscr{S}^{m}\left(R^{n} \times R^{n}\right)= & \text { the set of all elements of } \mathscr{S}^{m}\left([0, T] \times[0, T] \times R^{n} \times R^{n}\right) \\
& \text { which are independent of } t \text { and } s .
\end{aligned}
$$

If $A=\left[a_{i j}(t, s, x, \xi)\right]$ is an $N \times N$ matrix with each entry $a_{i j} \in \mathscr{S}^{m}([0, T]$ $\left.\times[0, T] \times R^{n} \times R^{n}\right)$, we still write $A \in \mathscr{S}^{m}\left([0, T] \times[0, T] \times R^{n} \times R^{n}\right)$. If each entry $a_{i j}$ belongs to $\mathscr{S}^{m}\left([0, T] \times R^{n} \times R^{n}\right)\left(\right.$ resp. $\left.\mathscr{S}^{m}\left(R^{n} \times R^{n}\right)\right)$, we also write $A \in \mathscr{S}^{m}\left([0, T] \times R^{n} \times R^{n}\right)$ (resp. $\left.\mathscr{S}^{m}\left(R^{n} \times R^{n}\right)\right)$. 
For $a(t, s, x, \xi) \in \mathscr{S}^{m}\left([0, T] \times[0, T] \times R^{n} \times R^{n}\right), a\left(t, s, x, D_{x}\right)$ denotes the pseudodifferential operator depending smoothly on $t$ and $s$ defined by

$$
a\left(t, s, x, D_{x}\right) f=(2 \pi)^{-n} \int_{R^{n}} a(t, s, x, \xi) e^{i x \cdot \xi} \hat{f}(\xi) d \xi,
$$

for each $f(x) \in C_{0}^{\infty}\left(R^{n}\right)$. We can also define $a\left(t, x, D_{x}\right)$ and $a\left(x, D_{x}\right)$ by $(0.13)$ if $a(t, x, \xi) \in \mathscr{S}^{m}\left([0, T] \times R^{n} \times R^{n}\right)$ and $a(x, \xi) \in \mathscr{S}^{m}\left(R^{n} \times R^{n}\right)$, respectively. When $A \in \mathscr{S}^{m}\left([0, T] \times[0, T] \times R^{n} \times R^{n}\right)$ is an $N \times N$ matrix, $A\left(t, s, x, D_{x}\right)$ is also defined by the formula $(0.13)$ with a $\mathscr{C}^{N}$ valued $f \in$ $C_{0}^{\infty}\left(R^{n}\right)$. In the same way, $A\left(t, x, D_{x}\right)$ and $A\left(x, D_{x}\right)$ can also be defined.

For $m \in R$, we write

$$
O P S^{m}\left([0, T] \times[0, T] \times R^{n}\right)=\text { the set of all } a\left(t, s, x, D_{x}\right)
$$
where $a(t, s, x, \xi) \in \mathscr{S}^{m}\left([0, T] \times[0, T] \times R^{n} \times R^{n}\right)$,

(0.16) $\operatorname{OPS}^{m}\left(R^{n}\right)=$ the set of all $a\left(x, D_{x}\right)$, where $a(x, \xi) \in \mathscr{S}^{m}\left(R^{n} \times R^{n}\right)$,

$$
O P S^{-\infty}\left([0, T] \times[0, T] \times R^{n}\right)=\bigcap_{m \in R} O P S^{m}\left([0, T] \times[0, T] \times R^{n}\right) .
$$

Similarly, $O P S^{-\infty}\left([0, T] \times R^{n}\right)$ and $O P S^{-\infty}\left(R^{n}\right)$ are also defined.

\section{Statement of the Main Result}

We first state the assumptions and the main result for the general form of equations (0.1), and then recap them for the special case of homogeneous isotropic media.

1.1. The general case. We note that all the coefficients of $(0.1)$ are real valued, and make the following assumptions.

$$
\begin{gathered}
C_{i j}^{\alpha \beta}(t, x) \in C^{\infty}\left([0, \infty) \times R^{n}\right) \text { and all the } \\
\text { derivatives of each } C_{i j}^{\alpha \beta} \text { are bounded on }[0, \infty) \times R^{n} . \\
C_{i j}^{\alpha \beta}(t, x)=C_{\alpha j}^{i \beta}(t, x)=C_{j i}^{\beta \alpha}(t, x), \\
\text { for all }(t, x) \in[0, \infty) \times R^{n} \\
\text { and every } \alpha, \beta, i \text { and } j .
\end{gathered}
$$

There is a positive constant $c_{0}$ such that

$$
\begin{aligned}
C_{i j}^{\alpha \beta}(t, x) \varepsilon_{\alpha i} \varepsilon_{\beta j} \geq c_{0} \varepsilon_{\alpha i} \varepsilon_{\alpha i}, & \text { for all }(t, x) \in[0, \infty) \times R^{n} \text { and } \\
& \text { every symmetric tensor } \varepsilon_{\alpha i},
\end{aligned}
$$

$D_{i j}^{\alpha}(t, x) \in C^{\infty}\left([0, \infty) \times R^{n}\right)$ and all the derivatives of each $D_{i j}^{\alpha}$ are bounded on $[0, \infty) \times R^{n}$,

$$
M_{i j}^{\alpha \beta}(t, s, x), N_{i j}^{\alpha}(t, s, x) \in C^{\infty}\left([0, \infty) \times[0, \infty) \times R^{n}\right),
$$

and all of their derivatives are bounded on $[0, \infty) \times[0, \infty) \times R^{n}$. 
Next we let $\lambda_{k}(t, x, \xi), k=1, \ldots, p$, represent the distinct eigenvalues of the matrix $\left[C_{i j}^{\alpha \beta}(t, x) \xi_{\alpha} \xi_{\beta}\right], \xi=\left(\xi_{1}, \ldots, \xi_{n}\right) \neq 0$, such that

$$
\lambda_{1}(t, x, \xi)<\cdots<\lambda_{p}(t, x, \xi) .
$$

We assume that there is a positive constant $c_{1}$ such that if $k \neq l$, for $k, l=1, \ldots, p$,

$$
\left|\lambda_{k}(t, x, \xi)-\lambda_{l}(t, x, \xi)\right| \geq c_{1}
$$

holds for all $(t, x) \in[0, \infty) \times R^{n}$ and $|\xi|=1$.

Under the above assumptions, it is easy to see that the algebraic multiplicity of each $\lambda_{k}$ is independent of $(t, x, \xi) \in[0, \infty) \times R^{n} \times\left(R^{n} \backslash\{0\}\right)$ and that

$$
\lambda_{1}(t, x, \xi) \geq \frac{1}{2} c_{0}|\xi|^{2},
$$

for all $(t, x, \xi) \in[0, \infty) \times R^{n} \times R^{n}$. We also have the following fact.

Lemma 1.1. For $k=1, \ldots, p, \lambda_{k}(t, x, \xi)$ is homogeneous of degree two in $\xi \neq 0$, and for each $(\alpha, \beta, \gamma) \in Z_{+} \times Z_{+}^{n} \times Z_{+}^{n}$ there is a positive constant $K_{\alpha \beta \gamma}$ such that

$$
\left|\partial_{t}^{\alpha} \partial_{x}^{\beta} \partial_{\xi}^{\gamma} \lambda_{k}(t, x, \xi)\right| \leq K_{\alpha \beta \gamma}|\xi|^{2-|\gamma|},
$$

for all $(t, x, \xi) \in[0, \infty) \times R^{n} \times\left(R^{n} \backslash\{0\}\right)$.

For the proof of (1.8), we use (1.1) and (1.6) to represent the projection operator associated with $\lambda_{k}(t, x, \xi)$ in terms of the Dunford integral. Then the projection operator is used to express $\lambda_{k}(t, x, \xi)$ through the trace formula. Since the argument is standard in the theory of perturbation of eigenvalues, we omit the details.

Next we set

$$
\mathscr{P}_{k}(t, x, \tau, \xi)=\tau^{2}-\lambda_{k}(t, x, \xi), \quad \text { for } k=1, \ldots, p .
$$

Definition 1.2. A bicharacteristic strip of $(0.1)$ is a solution $(t(s), x(s), \tau(s)$, $\xi(s))$ of

$$
\begin{gathered}
\frac{d x_{j}}{d s}=\frac{\partial}{\partial \xi_{j}} \mathscr{P}_{k}(t, x, \tau, \xi), \quad j=1, \ldots, n, \\
\frac{d t}{d s}=\frac{\partial}{\partial \tau} \mathscr{P}_{k}(t, x, \tau, \xi), \\
\frac{d \xi_{j}}{d s}=-\frac{\partial}{\partial x_{j}} \mathscr{P}_{k}(t, x, \tau, \xi), \quad j=1, \ldots, n, \\
\frac{d \tau}{d s}=-\frac{\partial}{\partial t} \mathscr{P}_{k}(t, x, \tau, \xi), \\
\mathscr{P}_{k}(t(s), x(s), \tau(s), \xi(s))=0 \text { and } \tau(s) \neq 0,
\end{gathered}
$$

for some $k=1, \ldots, p$.

If $\tau>0$, then the above system is equivalent to

$$
\frac{d x_{j}}{d t}=-\frac{\partial}{\partial \xi_{j}} \sqrt{\lambda_{k}(t, x, \xi)}, \quad j=1, \ldots, n,
$$




$$
\begin{gathered}
\frac{d \xi_{j}}{d t}=\frac{\partial}{\partial x_{j}} \sqrt{\lambda_{k}(t, x, \xi)}, \quad j=1, \ldots, n, \\
\frac{d \tau}{d t}=\frac{\partial}{\partial t} \sqrt{\lambda_{k}(t, x, \xi)}, \\
\mathscr{P}_{k}(t, x(t), \tau(t), \xi(t))=0,
\end{gathered}
$$

and if $\tau<0$, then it is equivalent to

$$
\begin{gathered}
\frac{d x_{j}}{d t}=\frac{\partial}{\partial \xi_{j}} \sqrt{\lambda_{k}(t, x, \xi)}, \quad j=1, \ldots, n, \\
\frac{d \xi_{j}}{d t}=-\frac{\partial}{\partial x_{j}} \sqrt{\lambda_{k}(t, x, \xi)}, \quad j=1, \ldots, n, \\
\frac{d \tau}{d t}=-\frac{\partial}{\partial t} \sqrt{\lambda_{k}(t, x, \xi)}, \\
\mathscr{P}_{k}(t, x(t), \tau(t), \xi(t))=0 .
\end{gathered}
$$

By virtue of Lemma 1.1, it is easy to see the following fact.

Lemma 1.3. Each bicharacteristic strip $(t, x(t), \tau(t), \xi(t))$ is defined globally in $t \in[0, \infty)$.

Definition 1.4. If $(t, x(t), \tau(t), \xi(t))$ is a bicharacteristic strip, then the curve $(t, x(t))$ in $[0, \infty) \times R^{n}$ is called a bicharacteristic curve.

We now state the main result.

Theorem 1.5. Suppose that all the above assumptions are satisfied and that $u$ is a solution of (0.1) and (0.2), where

$$
\left(u_{0}, u_{1}\right) \in H^{\nu}\left(R^{n}\right) \times H^{\nu-1}\left(R^{n}\right), \quad \text { for some } \nu \in R \text {. }
$$

If each bicharacteristic curve $(t, x(t))$ passing through $\left(t^{*}, x^{*}\right), t^{*}>0$, does not intersect the singular support of $u_{0}$ and $u_{1}$ at $t=0$, then there is a function $\phi(t, x) \in C_{0}^{\infty}\left(R^{n+1}\right)$ which is identically 1 in a neighborhood of $\left(t^{*}, x^{*}\right)$ and such that

$$
\phi u \in C^{\infty}\left(R ; H^{\nu+1}\left(R^{n}\right)\right) .
$$

Furthermore, if, in addition, $x^{*}$ does not belong to the singular support of $u_{0}$ and $u_{1}$, then

$$
\phi u \in C^{\infty}\left(R^{n+1}\right) .
$$

A few remarks are in order concerning the scope of the above theorem. The theorem does not imply that stationary singularities must appear. Even if all the above assumptions are satisfied, singular initial data do not necessarily generate stationary singularities. An interesting example in one space dimension was given in [10]. However, we can easily obtain a sufficient condition for the emergence of stationary singularities as a byproduct from the proof of the above theorem. Since the condition involves a technical tool, we will postpone its presentation: see Proposition 4.12. 
We note that (1.5) is the only assumption on the kernel functions for our result. The case of singular kernels is not addressed in this paper. A singular kernel can have a regularizing effect on solutions, and entirely different phenomena can occur. For various examples in one space dimension, the readers are again referred to [10]. It is known that some other condition on the memory kernel such as positive-definiteness enables the integral term to dissipate energy. This has been crucially used to obtain global solutions of nonlinear problems: see [14] for extensive references on this matter. This device does not improve the regularity of solutions, while it controls the growth of solutions if the kernel functions are smooth. In this paper, the asymptotic behavior of solutions is not an issue and such a condition, which is, in fact, consistent with real physics, does not help our analysis.

Next we shall outline the general strategy of the proof of the theorem. First of all, we need to make a precise statement on the existence and uniqueness of solutions to the Cauchy problem $(0.1)$ and $(0.2)$. We also need to investigate the domain of dependence of solutions, which is technically necessary in the process of the proof of Theorem 1.5. This will be done in $\S 2$. We then put the system $(0.1)$ in the following form:

$$
u_{t t}=L(t) u+\int_{0}^{t} \Phi(t, s) u(s) d s
$$

where $L(t)$ and $\Phi(t, s)$ stand for $n \times n$ matrices of differential operators of second order which depend smoothly on the parameters $t$ and $s$, and $u$ denotes the displacement vector with components $u_{i}, i=1, \ldots, n$. We employ MacCamy's trick to reduce the order of the integral term. In contrast to the case of one space dimension, the use of MacCamy's trick involves manipulation of some pseudodifferential operators. We put

$$
v=u+\int_{0}^{t} \widetilde{L}(t) \Phi(t, s) u(s) d s,
$$

where $\widetilde{L}(t)$ is a parametrix of $L(t)$ so that the operator $L(t) \widetilde{L}(t)-I$ is regularizing. $I$ is the $n \times n$ identity matrix.

Then $\widetilde{L}(t) \Phi(t, s)$ is an $n \times n$ matrix of pseudodifferential operators of order zero, and we solve for $u$, which can be represented by

$$
u=v+\int_{0}^{t} R(t, s) v(s) d s .
$$

Here $R(t, s)$ is an operator which can be formally obtained through the standard procedure for Volterra integral equations. But we have to prove that $R(t, s)$ is an $n \times n$ matrix of pseudodifferential operators of order zero which depend smoothly on $t$ and $s$. For the proof of this, a result of Beals [1] is a key element. The details are given in $\S 3$. After this, we write (1.26) in terms of $v$ so that

$$
\begin{aligned}
v_{t t}= & L v-\left(2 R_{t}(t, t)+R_{s}(t, t)\right) v-R(t, t) v_{t} \\
& -\int_{0}^{t} R_{t t}(t, s) v(s) d s+f,
\end{aligned}
$$

for some $f \in C^{\infty}\left([0, \infty) \times R^{n}\right)$. We then diagonalize $L(t)$ to treat the microlocal regularity of each component of $v$. Here, Hörmander's result on the 
propagation of singularities is crucial. After we establish the local regularity of $v$, we translate it into the local regularity of $u$ and complete the proof. The technical details are given in $\S 4$.

1.2. Homogeneous isotropic case. We will recap our main result in the context of a homogeneous isotropic medium in $R^{3}$. In this case, $(0.5)$ reduces to

$$
K_{i j}^{\alpha \beta}(t, x)=p(t)\left(\delta_{i j} \delta_{\alpha \beta}+\delta_{i \beta} \delta_{\alpha j}\right)+q(t) \delta_{i \alpha} \delta_{j \beta},
$$

where $\delta_{i j}$ is the Kronecker delta and $p(t), q(t)$ are given by

$$
\begin{gathered}
p(t)=\mu(0) \delta(t)+H(t) \frac{d}{d t} \mu(t), \\
q(t)=\lambda(0) \delta(t)+H(t) \frac{d}{d t} \lambda(t) .
\end{gathered}
$$

Here $\mu(t)$ and $\lambda(t)+\frac{2}{3} \mu(t)$ are associated with shear and volumetric deformation, respectively. By putting $\lambda=\lambda(0), \mu=\mu(0), \dot{\lambda}(t)=\frac{d}{d t} \lambda(t)$ and $\dot{\mu}(t)=\frac{d}{d t} \mu(t)$, the equations of motion $(0.1)$ reduce to

$$
\begin{aligned}
\frac{\partial^{2}}{\partial t^{2}} u^{i}(t, x)= & (\lambda+\mu) \frac{\partial}{\partial x_{i}} \frac{\partial}{\partial x_{j}} u^{j}(t, x)+\mu \frac{\partial^{2}}{\partial x_{j} \partial x_{j}} u^{i}(t, x) \\
& +\int_{0}^{t}(\dot{\lambda}(t-s)+\dot{\mu}(t-s)) \frac{\partial}{\partial x_{i}} \frac{\partial}{\partial x_{j}} u^{j}(s, x) d s \\
& +\int_{0}^{t} \dot{\mu}(t-s) \frac{\partial^{2}}{\partial x_{j} \partial x_{j}} u^{i}(s, x) d s, \quad i=1,2,3,
\end{aligned}
$$

where the mass density is taken to be 1 . Without the integral terms, these are the well-known equations of motion in linear elasticity for homogeneous isotropic media, where $\lambda, \mu$ are the Lamé constants, and $\frac{1}{3}(3 \lambda+2 \mu), \mu(3 \lambda+2 \mu) /(\lambda+\mu)$ are called the modulus of compression and Young's modulus, respectively. It is natural to assume that

$$
\mu>0, \quad 3 \lambda+2 \mu>0,
$$

from which the condition (1.3) follows. The condition (1.5) will also follow if suitable assumptions are made on $\dot{\lambda}(t)$ and $\dot{\mu}(t)$ in an obvious manner. The condition (1.4) is trivial. Here the matrix $\left[C_{i j}^{\alpha \beta}(t, x) \xi_{\alpha} \xi_{\beta}\right]$ becomes

$$
\left(\begin{array}{ccc}
\mu|\xi|^{2}+(\lambda+\mu) \xi_{1} \xi_{1}, & (\lambda+\mu) \xi_{1} \xi_{2}, & (\lambda+\mu) \xi_{1} \xi_{3} \\
(\lambda+\mu) \xi_{2} \xi_{1}, & \mu|\xi|^{2}+(\lambda+\mu) \xi_{2} \xi_{2}, & (\lambda+\mu) \xi_{2} \xi_{3} \\
(\lambda+\mu) \xi_{3} \xi_{1}, & (\lambda+\mu) \xi_{3} \xi_{2}, & \mu|\xi|^{2}+(\lambda+\mu) \xi_{3} \xi_{3}
\end{array}\right)
$$

This matrix has a simple eigenvalue $(\lambda+2 \mu)|\xi|^{2}$ and a double eigenvalue $\mu|\xi|^{2}$ for $|\xi|>0$. Therefore, the conditions (1.6) and (1.7) also follow from (1.34). Since the eigenvalues are independent of $t$ and $x$, the bicharacteristic strips given by (1.15) through (1.22) are all straight lines.

We now recap Theorem 1.5 in this special case. Let $\left(t^{*}, x^{*}\right), t^{*}>0$, be fixed. Then there are two cones in $[0, \infty) \times R^{3}$ with vertices at $\left(t^{*}, x^{*}\right)$. The first cone consists of family of straight lines given by

$$
x=x^{*}+\sqrt{\mu} \xi\left(t-t^{*}\right), \quad 0 \leq t \leq t^{*},|\xi|=1 .
$$


It is evident that this generates a cone as $\xi$ runs through the unit sphere $|\xi|=1$. The second cone is generated by

$$
x=x^{*}+\sqrt{2 \mu+\lambda} \xi\left(t-t^{*}\right), \quad 0 \leq t \leq t^{*},|\xi|=1 .
$$

If these two cones do not intersect the singular support of the initial data at $t=0$, then (1.24) follows.

\section{EXISTENCE, UNIQUENESS, AND DOMAIN OF DEPENDENCE}

The existence theorem is known in some particular function classes which include the function space of finite natural energy. A more general result on the existence of solutions is necessary to justify the statement of Theorem 1.5, and the domain of dependence of solutions is used in its proof. The existence theorems for an initial-boundary value problem can be found in [3] and [14], among others. Our existence theorem for the Cauchy problem is stated as follows.

Theorem 2.1. Let $\left(u_{0}, u_{1}\right) \in H^{\nu}\left(R^{n}\right) \times H^{\nu-1}\left(R^{n}\right)$, for some $\nu \in R$. Under the assumptions (1.1) through (1.5), there is a unique solution $u(t, x)$ of $(0.1)$ and $(0.2)$ in $C\left([0, \infty) ; H^{\nu}\left(R^{n}\right)\right) \cap C^{1}\left([0, \infty) ; H^{\nu-1}\left(R^{n}\right)\right)$. In fact, $\partial_{t}^{k} u \in$ $C\left([0, \infty) ; H^{\nu-k}\left(R^{n}\right)\right)$, for each $k \geq 1$.

Proof. Since all the coefficients in (0.1) are real, it is enough to consider only real-valued functions. We will adapt an argument for the symmetric hyperbolic system without an integral term; see [15]. Let us write $(0.1)$ as

$$
u_{t t}=L(t) u+\int_{0}^{t} \Phi(t, s) u(s) d s
$$

where $L(t)$ and $\Phi(t, s)$ are matrix differential operators of second order which depend smoothly on $t$ and $s$. We need pseudodifferential operators whose symbols are defined by

$$
\begin{aligned}
\Lambda^{m}(\xi) & =\left(1+|\xi|^{2}\right)^{m / 2}, \quad m \in R \\
\psi_{\varepsilon}(\xi) & =\left(1+\varepsilon|\xi|^{2}\right)^{-1 / 2}, \quad \varepsilon>0 .
\end{aligned}
$$

Then, we have

$$
\begin{array}{cc}
\Lambda^{m}\left(D_{x}\right) \in O P S^{m}\left(R^{n}\right), & m \in R \\
\psi_{\varepsilon}\left(D_{x}\right) \in O P S^{-1}\left(R^{n}\right), & \varepsilon>0
\end{array}
$$

From now on, the argument $D_{x}$ will be suppressed. We also note that these operators map real valued functions to real valued functions, and define, for $\varepsilon>0$,

so that

$$
G_{\varepsilon}(t) \in O P S^{0}\left([0, \infty) \times R^{n}\right),
$$




$$
N_{\varepsilon}(t, s) \in O P S^{0}\left([0, \infty) \times[0, \infty) \times R^{n}\right) .
$$

Here we have used the notation $(0.14)$ and $(0.15)$ with $[0, T]$ replaced by $[0, \infty)$. Let $\left(w_{0}, w_{1}\right) \in C_{0}^{\infty}\left(R^{n}\right) \times C_{0}^{\infty}\left(R^{n}\right)$ and consider the Cauchy problem with any fixed $\varepsilon>0$ :

$$
\begin{gathered}
w_{t t}^{\varepsilon}=G_{\varepsilon}(t) w^{\varepsilon}+\int_{0}^{t} N_{\varepsilon}(t, s) w^{\varepsilon}(s) d s, \\
w^{\varepsilon}(0)=w_{0}, \quad w_{t}^{\varepsilon}(0)=w_{1} .
\end{gathered}
$$

Since $G_{\varepsilon}(t)$ and $N_{\varepsilon}(t, s)$ are bounded linear operators from $H^{m}\left(R^{n}\right)$ into itself, for each $m \in R$, depending smoothly on $t$ and $s$, there is a unique solution $w^{\varepsilon}$ of $(2.11)$ and (2.12) such that

$$
w^{\varepsilon} \in C^{\infty}\left([0, \infty) ; H^{k}\left(R^{n}\right)\right), \quad \text { for all } k \in Z_{+} .
$$

Next we will suppress the superscript $\varepsilon$ and derive estimates. For this, we use the notation $\langle\cdot, \cdot\rangle$ which stands for the inner product in $L^{2}\left(R^{n}\right)$, and note that all the functions are $R^{n}$ valued. By applying $\Lambda^{\mu}$ to both sides of (2.11), we obtain

$$
\begin{aligned}
\left\langle\Lambda^{\mu} w_{t t}, \Lambda^{\mu} w_{t}\right\rangle= & \left\langle\Lambda^{\mu} G_{\varepsilon}(t) \Lambda^{-\mu} \Lambda^{\mu} w, \Lambda^{\mu} w_{t}\right\rangle \\
& +\left\langle\int_{0}^{t} \Lambda^{\mu} N_{\varepsilon}(t, s) w(s) d s, \Lambda^{\mu} w_{t}\right\rangle
\end{aligned}
$$

and

$$
\begin{aligned}
\left\langle\Lambda^{\mu} w_{t}, \Lambda^{\mu} w_{t t}\right\rangle= & \left\langle\left(\Lambda^{\mu} G_{\varepsilon}(t) \Lambda^{-\mu}\right)^{*} \Lambda^{\mu} w_{t}, \Lambda^{\mu} w\right\rangle \\
& +\left\langle\Lambda^{\mu} w_{t}, \int_{0}^{t} \Lambda^{\mu} N_{\varepsilon}(t, s) w(s) d s\right\rangle,
\end{aligned}
$$

where $\left(\Lambda^{\mu} G_{\varepsilon}(t) \Lambda^{-\mu}\right)^{*}$ denotes the adjoint of $\Lambda^{\mu} G_{\varepsilon}(t) \Lambda^{-\mu}$. We then define

$$
H_{\varepsilon}(t)=\Lambda^{\mu} G_{\varepsilon}(t) \Lambda^{-\mu}-\left(\Lambda^{\mu} G_{\varepsilon}(t) \Lambda^{-\mu}\right)^{*}
$$

so that

$$
\left\{H_{\varepsilon}(t): 0<\varepsilon \leq 1\right\} \text { is a bounded subset of } \operatorname{OPS}^{1}\left([0, \infty) \times R^{n}\right),
$$

since $\left\{G_{\varepsilon}(t): 0<\varepsilon \leq 1\right\}$ is a bounded subset of $O P S^{2}\left([0, \infty) \times R^{n}\right)$ and the principal symbol of $G_{\varepsilon}(t)$ is symmetric. Using this, we add (2.14) and (2.15) to obtain

$$
\begin{aligned}
\frac{d}{d t}\left(\left\langle\Lambda^{\mu} w_{t}, \Lambda^{\mu} w_{t}\right\rangle-\left\langle\Lambda^{\mu} G_{\varepsilon}(t) \Lambda^{-\mu} \Lambda^{\mu} w, \Lambda^{\mu} w\right\rangle\right) \\
=-\left\langle\Lambda^{\mu} w_{t},\left(H_{\varepsilon}(t)\right)^{*} \Lambda^{\mu} w\right\rangle-\left\langle\Lambda^{\mu} G_{\varepsilon t}(t) \Lambda^{-\mu} \Lambda^{\mu} w, \Lambda^{\mu} w\right\rangle \\
\quad+2\left\langle\int_{0}^{t} \Lambda^{\mu} N_{\varepsilon}(t, s) w(s) d s, \Lambda^{\mu} w_{t}(t)\right\rangle
\end{aligned}
$$

where $\left(H_{\varepsilon}(t)\right)^{*}$ is the adjoint of $H_{\varepsilon}(t)$ and $G_{\varepsilon t}(t)$ is the derivative of $G_{\varepsilon}(t)$ in $t$.

By virtue of the assumptions (1.1) through (1.4), we can apply Gärding's inequality to the operator $\Lambda^{\mu} L(t) \Lambda^{-\mu}$. Hence there are positive constants $\alpha_{1}$ and $\alpha_{2}$ such that

$$
-\left\langle\Lambda^{\mu} L(t) \Lambda^{-\mu} \phi, \phi\right\rangle \geq \alpha_{1}\|\phi\|_{H^{1}\left(R^{n}\right)}^{2}-\alpha_{2}\|\phi\|_{L^{2}\left(R^{n}\right)}^{2},
$$


for all $R^{n}$ valued $\phi \in C_{0}^{\infty}\left(R^{n}\right)$ and all $t \geq 0$. Since

$$
\Lambda^{\mu} G_{\varepsilon}(t) \Lambda^{-\mu}=\psi_{\varepsilon} \Lambda^{\mu} L(t) \Lambda^{-\mu} \psi_{\varepsilon},
$$

it follows from (2.19) that

$$
-\left\langle\Lambda^{\mu} G_{\varepsilon}(t) \Lambda^{-\mu} \Lambda^{\mu} w, \Lambda^{\mu} w\right\rangle \geq \alpha_{1}\left\|\psi_{\varepsilon} \Lambda^{\mu} w\right\|_{H^{1}\left(R^{n}\right)}^{2}-\alpha_{2}\left\|\psi_{\varepsilon} \Lambda^{\mu} w\right\|_{L^{2}\left(R^{n}\right)}^{2} .
$$

Next, by means of the identity

$$
H_{\varepsilon}(t)^{*}=\psi_{\varepsilon}\left(\left(\Lambda^{\mu} L(t) \Lambda^{-\mu}\right)^{*}-\left(\Lambda^{\mu} L(t) \Lambda^{-\mu}\right)\right) \psi_{\varepsilon},
$$

we can derive

$$
\left|\left\langle\Lambda^{\mu} w_{t},\left(H_{\varepsilon}(t)\right)^{*} \Lambda^{\mu} w\right\rangle\right| \leq \alpha_{3}\left(\left\|\psi_{\varepsilon} \Lambda^{\mu} w_{t}\right\|_{L^{2}\left(R^{n}\right)}^{2}+\left\|\psi_{\varepsilon} \Lambda^{\mu} w\right\|_{H^{1}\left(R^{n}\right)}^{2}\right),
$$

where $\alpha_{3}$ is a positive constant independent of $\varepsilon$ and $t$. We also use the identity

$$
\Lambda^{\mu} G_{\varepsilon t}(t) \Lambda^{-\mu}=\psi_{\varepsilon} \Lambda^{\mu} L_{t}(t) \Lambda^{-\mu} \psi_{\varepsilon}
$$

to obtain

$$
\left|\left\langle\Lambda^{\mu} G_{\varepsilon t}(t) \Lambda^{-\mu} \Lambda^{\mu} w, \Lambda^{\mu} w\right\rangle\right| \leq \alpha_{4}\left\|\psi_{\varepsilon} \Lambda^{\mu} w\right\|_{H^{1}\left(R^{n}\right)}^{2},
$$

where $\alpha_{4}$ is a positive constant independent of $\varepsilon$ and $t$. It now follows from (2.18) and the above estimate that

$$
\begin{aligned}
\left\|\Lambda^{\mu} w_{t}(t)\right\|_{L^{2}\left(R^{n}\right)}^{2}+\alpha_{1}\left\|\psi_{\varepsilon} \Lambda^{\mu} w(t)\right\|_{H^{1}\left(R^{n}\right)}^{2} & \\
\leq & \alpha_{2}\left\|\psi_{\varepsilon} \Lambda^{\mu} w(t)\right\|_{L^{2}\left(R^{n}\right)}^{2}+\alpha_{5}\left(\left\|\Lambda^{\mu} w_{0}\right\|_{H^{1}\left(R^{n}\right)}^{2}+\left\|\Lambda^{\mu} w_{1}\right\|_{L^{2}\left(R^{n}\right)}^{2}\right) \\
& +\alpha_{6} \int_{0}^{t}\left(\left\|\psi_{\varepsilon} \Lambda^{\mu} w(s)\right\|_{H^{1}\left(R^{n}\right)}^{2}+\left\|\psi_{\varepsilon} \Lambda^{\mu} w_{s}(s)\right\|_{L^{2}\left(R^{n}\right)}^{2}\right) d s \\
& +2 \int_{0}^{t}\left\langle\int_{0}^{s} \Lambda^{\mu} N_{\varepsilon}(s, \eta) w(\eta) d \eta, \Lambda^{\mu} w_{s}(s)\right\rangle d s
\end{aligned}
$$

for all $t \geq 0$ and all $0<\varepsilon \leq 1$, where $\alpha_{5}$ and $\alpha_{6}$ are positive constants. Now we observe that

$$
\begin{aligned}
\int_{0}^{t}\langle & \left.\int_{0}^{s} \Lambda^{\mu} N_{\varepsilon}(s, \eta) w(\eta) d \eta, \Lambda^{\mu} w_{s}(s)\right\rangle d s \\
= & \left\langle\int_{0}^{t} \Lambda^{\mu} N_{\varepsilon}(t, \eta) w(\eta) d \eta, \Lambda^{\mu} w(t)\right\rangle \\
& -\int_{0}^{t}\left\langle\int_{0}^{s} \Lambda^{\mu}\left(\partial_{s} N_{\varepsilon}(s, \eta)\right) w(\eta) d \eta, \Lambda^{\mu} w(s)\right\rangle d s \\
& -\int_{0}^{t}\left\langle\Lambda^{\mu} N_{\varepsilon}(s, s) w(s), \Lambda^{\mu} w(s)\right\rangle d s .
\end{aligned}
$$

Using the identity

$$
\Lambda^{\mu} N_{\varepsilon}(t, \eta)=\psi_{\varepsilon} \Lambda^{\mu} \Phi(t, \eta) \Lambda^{-\mu} \psi_{\varepsilon} \Lambda^{\mu},
$$

and Hölder's inequality, we can estimate each term of (2.26) so that

$$
\begin{aligned}
\mid \int_{0}^{t}\langle & \left\langle\int_{0}^{s} \Lambda^{\mu} N_{\varepsilon}(s, \eta) w(\eta) d \eta, \Lambda^{\mu} w_{s}(s)\right\rangle d s \mid \\
\leq & \frac{1}{2} \alpha_{1}\left\|\psi_{\varepsilon} \Lambda^{\mu} w(t)\right\|_{H^{1}\left(R^{n}\right)}^{2}+\alpha_{7} t \int_{0}^{t}\left\|\psi_{\varepsilon} \Lambda^{\mu} w(s)\right\|_{H^{1}\left(R^{n}\right)}^{2} d s \\
& +\alpha_{8} \int_{0}^{t}\left\|\psi_{\varepsilon} \Lambda^{\mu} w(s)\right\|_{H^{1}\left(R^{n}\right)}^{2} d s+\alpha_{9} t^{2} \int_{0}^{t}\left\|\psi_{\varepsilon} \Lambda^{\mu} w(s)\right\|_{H^{1}\left(R^{n}\right)}^{2} d s
\end{aligned}
$$


where $\alpha_{7}, \alpha_{8}$ and $\alpha_{9}$ are positive constants independent of $\varepsilon$ and $t$. We also find that

$$
\begin{aligned}
\left\|\psi_{\varepsilon} \Lambda^{\mu} w(t)\right\|_{L^{2}\left(R^{n}\right)}^{2} \leq & \left\|\psi_{\varepsilon} \Lambda^{\mu} w_{0}\right\|_{L^{2}\left(R^{n}\right)}^{2} \\
& +\int_{0}^{t}\left\{\left\|\psi_{\varepsilon} \Lambda^{\mu} w_{s}(s)\right\|_{L^{2}\left(R^{n}\right)}^{2}+\left\|\psi_{\varepsilon} \Lambda^{\mu} w(s)\right\|_{L^{2}\left(R^{n}\right)}^{2}\right\} d s,
\end{aligned}
$$

for all $t \geq 0$. Combining (2.6), (2.25), (2.28), and (2.29), we resort to Gronwall's inequality and put back the superscript $\varepsilon$ to obtain

$$
\begin{aligned}
& \left\|\psi_{\varepsilon} w^{\varepsilon}(t)\right\|_{H^{\mu+1}\left(R^{n}\right)}^{2}+\left\|w_{t}^{\varepsilon}(t)\right\|_{H^{\mu}\left(R^{n}\right)}^{2} \\
& \quad \leq \alpha(\mu, T)\left(\left\|w_{0}\right\|_{H^{\mu+1}\left(R^{n}\right)}^{2}+\left\|w_{1}\right\|_{H^{\mu}\left(R^{n}\right)}^{2}\right),
\end{aligned}
$$

for all $t \in[0, T]$ and all $\varepsilon \in(0,1]$. Here $\alpha(\mu, T)$ is a positive constant which depends on $\mu$ and $T>0$, but is independent of $\varepsilon$. Now fix any $T>0$. By virtue of (2.30), we can extract a sequence $\left\{w^{\varepsilon}(t)\right\}$ such that

$$
\begin{aligned}
\psi_{\varepsilon} w^{\varepsilon} \rightarrow w & \text { weak } * \text { in } L^{\infty}\left(0, T ; H^{\mu+1}\left(R^{n}\right)\right), \\
w_{t}^{\varepsilon} \rightarrow w_{t} & \text { weak } * \text { in } L^{\infty}\left(0, T ; H^{\mu}\left(R^{n}\right)\right),
\end{aligned}
$$

for every $\mu \in R$, for some $R^{n}$ valued function $w(t, x)$. It is easy to see that $w$ satisfies

$$
\begin{gathered}
w_{t t}=L(t) w+\int_{0}^{t} \Phi(t, s) w(s) d s \\
w(0)=w_{0}, \quad w_{t}(0)=w_{1} .
\end{gathered}
$$

It follows from (2.33) that

$$
w \in C^{\infty}\left([0, T] ; H^{\mu}\left(R^{n}\right)\right),
$$

for every $\mu \in R$. Hence, we have established the existence of smooth solutions when the initial data are in $C_{0}^{\infty}\left(R^{n}\right)$. For two smooth solutions $w^{1}$ and $w^{2}$, we have the following estimate

$$
\begin{aligned}
& \left\|w^{1}(t)-w^{2}(t)\right\|_{H^{\mu+1}\left(R^{n}\right)}^{2}+\left\|w_{t}^{1}(t)-w_{t}^{2}(t)\right\|_{H^{\mu}\left(R^{n}\right)}^{2} \\
& \quad \leq \alpha(\mu, T)\left(\left\|w^{1}(0)-w^{2}(0)\right\|_{H^{\mu+1}\left(R^{n}\right)}^{2}+\left\|w_{t}^{1}(0)-w_{t}^{2}(0)\right\|_{H^{\mu}\left(R^{n}\right)}^{2}\right),
\end{aligned}
$$

for all $t \in[0, T]$ and each $\mu \in R$. This can be obtained through a similar procedure as above, but without using $\psi_{\varepsilon}$. Now suppose that $\left(u_{0}, u_{1}\right) \in$ $H^{\nu}\left(R^{n}\right) \times H^{\nu-1}\left(R^{n}\right)$ is given. Then, we can approximate $\left(u_{0}, u_{1}\right)$ by a sequence in $C_{0}^{\infty}\left(R^{n}\right) \times C_{0}^{\infty}\left(R^{n}\right)$ which converges to $\left(u_{0}, u_{1}\right)$ in $H^{\nu}\left(R^{n}\right) \times H^{\nu-1}\left(R^{n}\right)$. For each approximate data, we obtain a smooth solution. By means of (2.36), we obtain a true solution $u$ in $C\left([0, T] ; H^{\nu}\left(R^{n}\right)\right) \cap C^{1}\left([0, T] ; H^{\nu-1}\left(R^{n}\right)\right)$. It follows from (2.1) that

$$
\partial_{t}^{k} u \in C\left([0, T] ; H^{\nu-k}\left(R^{n}\right)\right),
$$

for each $k \geq 1$. Here $T$ can be arbitrarily large. Uniqueness follows by repetition of the above argument, and the proof is complete.

Next we state the domain of dependence of solutions. This is not of a sharp form, but it is enough for our purpose and the proof is simpler. Let us fix any $t_{0}>0$ and $x_{0} \in R^{n}$, and define for $0 \leq t \leq t_{0}, \varepsilon>0$ and $\eta>0$,

$$
\Omega(t)=\left\{x \in R^{n}:\left\|x-x_{0}\right\| \leq \varepsilon+2 \eta\left(t_{0}-t\right)\right\} .
$$


Then $\Omega(t)$ is a closed $n$-dimensional ball for each $t$, and $\bigcup_{0 \leq t \leq t_{0}}\{(t, \Omega(t))\}$ is a truncated cone in $R^{n+1}$. For the time being, we suppress the dependence of $\Omega(t)$ on $\varepsilon>0$ and $\eta>0$.

Theorem 2.2. Let $u$ be a solution of $(0.1)$ and $(0.2)$ with $\left(u_{0}, u_{1}\right) \in H^{\nu}\left(R^{n}\right) \times$ $H^{\nu-1}\left(R^{n}\right)$, for some $\nu \in R$, under the assumptions (1.1) through (1.5). There is a positive constant $\eta$ depending only on the coefficients of $(0.1)$ such that if $\Omega(0)$ is disjoint from supp $u_{0} \cup \operatorname{supp} u_{1}$ for some $\varepsilon>0$, then $u=0$ in the set $\bigcup_{0 \leq t \leq t_{0}}\{(t, \Omega(t))\}$.

Proof. We shall proceed in the spirit of [4], which discussed the equations of homogeneous anisotropic elasticity. On account of the memory term, our calculations are more involved. We rewrite $(0.1)$ as

$$
\begin{aligned}
u_{t t}^{i}= & \left(C_{i j}^{\alpha \beta} u_{\beta}^{j}\right)_{\alpha}+E_{i j}^{\alpha} u_{\alpha}^{j} \\
& +\int_{0}^{t}\left(M_{i j}^{\alpha \beta}(t, s) u_{\beta}^{j}(s)\right)_{\alpha} d s+\int_{0}^{t} K_{i j}^{\alpha}(t, s) u_{\alpha}^{j}(s) d s,
\end{aligned}
$$

where the subscripts $\alpha$ and $\beta$ denote the partial differentiation in $x_{\alpha}$ and $x_{\beta}$, respectively. Here, $E_{i j}^{\alpha}$ and $K_{i j}^{\alpha}$ are derived from the coefficients of $(0.1)$ in an obvious manner. Let us assume

$$
\left(u_{0}, u_{1}\right) \in C_{0}^{\infty}\left(R^{n}\right) \times C_{0}^{\infty}\left(R^{n}\right),
$$

so that the solution is smooth. For the above fixed $t_{0}>0$ and $x_{0} \in R^{n}$, we put for $0 \leq t \leq t_{0}, \varepsilon>0$ and $\eta>0$,

$$
\mathscr{E}(t)=\frac{1}{2} \int_{\Omega(t)}\left(u_{t}^{i} u_{t}^{i}+C_{i j}^{\alpha \beta} u_{\alpha}^{i} u_{\beta}^{j}\right) d x .
$$

We then differentiate $\mathscr{E}(t)$ in $t$ to obtain

$$
\begin{aligned}
\frac{d \mathscr{E}(t)}{d t}= & \int_{\Omega(t)}\left(u_{t}^{i} u_{t t}^{i}+C_{i j}^{\alpha \beta} u_{\alpha}^{i} u_{\beta t}^{j}\right) d x+\frac{1}{2} \int_{\Omega(t)} C_{i j t}^{\alpha \beta} u_{\alpha}^{i} u_{\beta}^{j} d x \\
& -\eta \int_{\partial \Omega(t)}\left(u_{t}^{i} u_{t}^{i}+C_{i j}^{\alpha \beta} u_{\alpha}^{i} u_{\beta}^{j}\right) d x \\
= & \int_{\Omega(t)}\left(u_{t}^{i} C_{i j}^{\alpha \beta} u_{\beta}^{j}\right)_{\alpha} d x+\int_{\Omega(t)} u_{t}^{i} E_{i j}^{\alpha} u_{\alpha}^{j} d x \\
& +\int_{\Omega(t)} u_{t}^{i}(t)\left\{\int_{0}^{t}\left(M_{i j}^{\alpha \beta}(t, s) u_{\beta}^{j}(s)\right)_{\alpha} d s\right\} d x \\
& +\int_{\Omega(t)} u_{t}^{i}(t)\left\{\int_{0}^{t} K_{i j}^{\alpha}(t, s) u_{\alpha}^{j}(s) d s\right\} d x \\
& +\frac{1}{2} \int_{\Omega(t)} C_{i j t}^{\alpha \beta} u_{\alpha}^{i} u_{\beta}^{j} d x-\eta \int_{\partial \Omega(t)}\left(u_{t}^{i} u_{t}^{i}+C_{i j}^{\alpha \beta} u_{\alpha}^{i} u_{\beta}^{j}\right) d x .
\end{aligned}
$$


By integration by parts, we have

$$
\begin{aligned}
\frac{d \mathscr{E}(t)}{d t}= & -\eta \int_{\partial \Omega(t)}\left(u_{t}^{i} u_{t}^{i}+C_{i j}^{\alpha \beta} u_{\alpha}^{i} u_{\beta}^{j}\right) d x \\
& +\int_{\partial \Omega(t)} \zeta_{\alpha} u_{t}^{i} C_{i j}^{\alpha \beta} u_{\beta}^{j} d x+\int_{\Omega(t)} u_{t}^{i} E_{i j}^{\alpha} u_{\alpha}^{j} d x \\
& +\int_{\partial \Omega(t)} \zeta_{\alpha} u_{t}^{i}(t)\left\{\int_{0}^{t} M_{i j}^{\alpha \beta}(t, s) u_{\beta}^{j}(s) d s\right\} d x \\
& -\int_{\Omega(t)} u_{\alpha t}^{i}(t)\left\{\int_{0}^{t} M_{i j}^{\alpha \beta}(t, s) u_{\beta}^{j}(s) d s\right\} d x \\
& +\int_{\Omega(t)} u_{t}^{i}(t)\left\{\int_{0}^{t} K_{i j}^{\alpha}(t, s) u_{\alpha}^{j}(s) d s\right\} d x \\
& +\frac{1}{2} \int_{\Omega(t)} C_{i j t}^{\alpha \beta} u_{\alpha}^{i} u_{\beta}^{j} d x,
\end{aligned}
$$

where $\zeta=\left(\zeta_{1}, \ldots, \zeta_{n}\right)$ is the outward unit normal vector on $\partial \Omega(t)$ with respect to the $x$ variables. In fact, $\zeta=\left(x-x_{0}\right) /\left\|x-x_{0}\right\|$. We will estimate each integral of (2.41). In the following estimates, $k_{i}$ 's denote positive constants which depend only on the coefficients of (2.38). First we observe the following identity as in [4] by using (1.2) and (1.3):

$$
\begin{aligned}
& -\frac{1}{2} C_{i j}^{\alpha \beta} u_{\alpha}^{i} u_{\beta}^{j}+\frac{1}{\eta} \zeta_{\alpha} u_{t}^{i} C_{i j}^{\alpha \beta} u_{\beta}^{j} \\
& \quad=-\frac{1}{2} C_{i j}^{\alpha \beta}\left(u_{\alpha}^{i}-\frac{1}{\eta} \zeta_{\alpha} u_{t}^{i}\right)\left(u_{\beta}^{j}-\frac{1}{\eta} \zeta_{\beta} u_{t}^{j}\right)+\frac{1}{2 \eta^{2}} C_{i j}^{\alpha \beta} \zeta_{\alpha} \zeta_{\beta} u_{t}^{i} u_{t}^{j} \\
& \quad \leq \frac{1}{2 \eta^{2}} C_{i j}^{\alpha \beta} \zeta_{\alpha} \zeta_{\beta} u_{t}^{i} u_{t}^{j}
\end{aligned}
$$

and thus,

$$
\begin{aligned}
& -\eta \int_{\partial \Omega(t)} C_{i j}^{\alpha \beta} u_{\alpha}^{i} u_{\beta}^{j} d x+\int_{\partial \Omega(t)} \zeta_{\alpha} u_{t}^{i} C_{i j}^{\alpha \beta} u_{\beta}^{j} d x \\
& \quad \leq \frac{k_{1}}{\eta} \int_{\partial \Omega(t)} u_{t}^{i} u_{t}^{i} d x-\frac{\eta}{2} \int_{\partial \Omega(t)} C_{i j}^{\alpha \beta} u_{\alpha}^{i} u_{\beta}^{j} d x .
\end{aligned}
$$

For the fourth integral $(2.41)$, we integrate in $t$ over $[0, h]$, for $0 \leq h \leq t_{0}$, so that

$$
\begin{aligned}
& \int_{0}^{h}\left(\int_{\partial \Omega(t)} \zeta_{\alpha} u_{t}^{i}(t)\left\{\int_{0}^{t} M_{i j}^{\alpha \beta}(t, s) u_{\beta}^{j}(s) d s\right\} d x\right) d t \\
& \quad \leq \gamma \int_{0}^{h} \int_{\partial \Omega(t)} u_{t}^{i}(t) u_{t}^{i}(t) d x d t \\
& \quad+\frac{k_{2} h}{\gamma} \int_{0}^{h}\left\{\int_{\partial \Omega(t)} \int_{0}^{t} u_{\beta}^{j}(s) u_{\beta}^{j}(s) d s d x\right\} d t
\end{aligned}
$$


for any $\gamma>0$. The last integral of $(2.44)$ can be estimated as follows:

$$
\begin{aligned}
\int_{0}^{h} & \left\{\int_{\partial \Omega(t)} \int_{0}^{t} u_{\beta}^{j}(s) u_{\beta}^{j}(s) d s d x\right\} d t \\
& =\int_{0}^{h}\left\{\int_{0}^{t} \int_{\partial \Omega(t)} u_{\beta}^{j}(s) u_{\beta}^{j}(s) d x d s\right\} d t, \\
& \text { by putting } \rho=\varepsilon+2 \eta\left(t_{0}-t\right), \\
& =\frac{1}{2 \eta} \int_{\varepsilon+2 \eta\left(t_{0}-h\right)}^{\varepsilon+2 \eta t_{0}} \int_{0}^{t_{0}+(\varepsilon-\rho) /(2 \eta)} \int_{\left\|x-x_{0}\right\|=\rho} u_{\beta}^{j}(s) u_{\beta}^{j}(s) d x d s d \rho,
\end{aligned}
$$

by changing the order of integration,

$$
\begin{aligned}
& \leq \frac{1}{2 \eta} \int_{0}^{h} \int_{0}^{\varepsilon+2 \eta\left(t_{0}-s\right)} \int_{\left\|x-x_{0}\right\|=\rho} u_{\beta}^{j}(s) u_{\beta}^{j}(s) d x d \rho d s \\
& =\frac{1}{2 \eta} \int_{0}^{h} \int_{\Omega(t)} u_{\beta}^{j}(t) u_{\beta}^{j}(t) d x d t .
\end{aligned}
$$

For the fifth integral of (2.41), we integrate in $t$ over $[0, h]$, for $0 \leq h \leq t_{0}$, and use integration by parts to obtain

$$
\begin{aligned}
-\int_{0}^{h} & \int_{\Omega(t)} u_{\alpha t}^{i}(t)\left\{\int_{0}^{t} M_{i j}^{\alpha \beta}(t, s) u_{\beta}^{j}(s) d s\right\} d x d t \\
= & -\int_{\Omega(h)} u_{\alpha}^{i}(h)\left\{\int_{0}^{h} M_{i j}^{\alpha \beta}(h, s) u_{\beta}^{j}(s) d s\right\} d x \\
& +\int_{0}^{h} \int_{\Omega(t)} u_{\alpha}^{i}(t) M_{i j}^{\alpha \beta}(t, t) u_{\beta}^{j}(t) d x d t \\
& +\int_{0}^{h} \int_{\Omega(t)} u_{\alpha}^{i}(t)\left\{\int_{0}^{t} M_{i j t}^{\alpha \beta}(t, s) u_{\beta}^{j}(s) d s\right\} d x d t \\
& -2 \eta \int_{0}^{h} \int_{\partial \Omega(t)} u_{\alpha}^{i}(t)\left\{\int_{0}^{t} M_{i j}^{\alpha \beta}(t, s) u_{\beta}^{j}(s) d s\right\} d x d t \\
\leq & \gamma \int_{\Omega(h)} u_{\alpha}^{i}(h) u_{\alpha}^{i}(h) d x+\gamma \int_{0}^{h} \int_{\partial \Omega(t)} u_{\alpha}^{i}(t) u_{\alpha}^{i}(t) d x d t \\
& +\left(\frac{k_{3} h}{\gamma}+k_{4}+k_{5} h^{2}+\frac{\eta k_{6} h}{\gamma}\right) \int_{0}^{h} \int_{\Omega(t)} u_{\alpha}^{i}(t) u_{\alpha}^{i}(t) d x d t,
\end{aligned}
$$

for any $\gamma>0$, where $(2.45)$ has been used. It is easy to see that (2.47)

$$
\begin{aligned}
& \int_{0}^{h}\{\text { the third integral }+ \text { the sixth integral } \\
& \qquad+ \text { the seventh integral of }(2.41)\} d t \\
& \leq k_{7} \int_{0}^{h} \int_{\Omega(t)} u_{t}^{i}(t) u_{t}^{i}(t) d x d t+\left(k_{8}+k_{9} h^{2}\right) \int_{0}^{h} \int_{\Omega(t)} u_{\alpha}^{i}(t) u_{\alpha}^{i}(t) d x d t .
\end{aligned}
$$

Combining the above estimates and calling forth the dependence of $\Omega(t)$ on 
$\varepsilon>0$, we arrive at

$$
\begin{aligned}
\mathscr{E}(h, \varepsilon) \leq & \mathscr{E}(0, \varepsilon)-\eta \int_{0}^{h} \int_{\partial \Omega(t, \varepsilon)} u_{t}^{i}(t) u_{t}^{i}(t) d x d t \\
& -\frac{\eta}{2} \int_{0}^{h} \int_{\partial \Omega(t, \varepsilon)} C_{i j}^{\alpha \beta} u_{\alpha}^{i}(t) u_{\beta}^{j}(t) d x d t \\
& +\left(\gamma+\frac{k_{1}}{\eta}\right) \int_{0}^{h} \int_{\partial \Omega(t, \varepsilon)} u_{t}^{i}(t) u_{t}^{i}(t) d x d t \\
& +\left(\frac{k_{2} h}{2 \gamma \eta}+\frac{k_{3} h}{\gamma}+k_{4}+k_{5} h^{2}+\frac{\eta k_{6} h}{\gamma}+k_{8}+k_{9} h^{2}\right) \\
& \times \int_{0}^{h} \int_{\Omega(t, \varepsilon)} u_{\alpha}^{i}(t) u_{\alpha}^{i}(t) d x d t \\
& +\gamma \int_{\Omega(h, \varepsilon)} u_{\alpha}^{i}(h) u_{\alpha}^{i}(h) d x+\gamma \int_{0}^{h} \int_{\partial \Omega(t, \varepsilon)} u_{\alpha}^{i}(t) u_{\alpha}^{i}(t) d x d t \\
& +k_{7} \int_{0}^{h} \int_{\Omega(t, \varepsilon)} u_{t}^{i}(t) u_{t}^{i}(t) d x d t,
\end{aligned}
$$

for every $\gamma>0, \eta>0,0 \leq h \leq t_{0}$ and $\varepsilon>0$. Next let us choose $\eta>0$ such that

$$
\frac{k_{1}}{\eta}<\frac{1}{4} \eta
$$

Now $t_{0}, x_{0}$ and $\eta$ are fixed. Suppose that there are $0<\varepsilon_{1}<\varepsilon_{2}$ such that

$$
\Omega\left(0, \varepsilon_{2}\right) \cap\left\{\operatorname{supp} u_{0} \cup \operatorname{supp} u_{1}\right\} \quad \text { is empty. }
$$

By virtue of Korn's inequality with the aid of (1.1), (1.2) and (1.3), there are positive constants $d_{1}$ and $d_{2}$ such that

$$
\begin{aligned}
& \int_{\varepsilon_{1}}^{\varepsilon_{2}} \int_{\partial \Omega(t, \varepsilon)} C_{i j}^{\alpha \beta} u_{\alpha}^{i}(t) u_{\beta}^{j}(t) d x d \varepsilon \\
& \quad \geq d_{1} \int_{\varepsilon_{1}}^{\varepsilon_{2}} \int_{\partial \Omega(t, \varepsilon)} u_{\alpha}^{i}(t) u_{\alpha}^{i}(t) d x d \varepsilon-d_{2} \int_{\varepsilon_{1}}^{\varepsilon_{2}} \int_{\partial \Omega(t, \varepsilon)} u^{i}(t) u^{i}(t) d x d \varepsilon,
\end{aligned}
$$

for all $0 \leq t \leq t_{0}$, and

$$
\begin{aligned}
& \int_{\Omega(t, \varepsilon)} C_{i j}^{\alpha \beta} u_{\alpha}^{i}(t) u_{\beta}^{j}(t) d x \\
& \quad \geq d_{1} \int_{\Omega(t, \varepsilon)} u_{\alpha}^{i}(t) u_{\alpha}^{i}(t) d x-d_{2} \int_{\Omega(t, \varepsilon)} u^{i}(t) u^{i}(t) d x,
\end{aligned}
$$

for all $0 \leq t \leq t_{0}$ and $\varepsilon_{1} \leq \varepsilon \leq \varepsilon_{2}$. Since

$$
u^{i}(t, x)=\int_{0}^{t} u_{t}^{i}(s, x) d s
$$

for all $x \in \Omega\left(0, \varepsilon_{2}\right)$, we can derive

$$
\int_{\Omega(t, \varepsilon)} u^{i}(t) u^{i}(t) d x \leq t \int_{0}^{t} \int_{\Omega(s, \varepsilon)} u_{t}^{i}(s) u_{t}^{i}(s) d x d s,
$$


for all $0 \leq t \leq t_{0}$ and $\varepsilon_{1} \leq \varepsilon \leq \varepsilon_{2}$. It also follows that

$$
\begin{aligned}
& \int_{\varepsilon_{1}}^{\varepsilon_{2}} \int_{\partial \Omega(t, \varepsilon)} u^{i}(t) u^{i}(t) d x d \varepsilon \leq t \int_{\varepsilon_{1}}^{\varepsilon_{2}} \int_{\partial \Omega(t, \varepsilon)} \int_{0}^{t} u_{t}^{i}(s) u_{t}^{i}(s) d s d x d \varepsilon \\
& \quad \leq t \int_{0}^{t} \int_{\varepsilon_{1}}^{\varepsilon_{2}} \int_{\partial \Omega(s, \varepsilon)} u_{t}^{i}(s) u_{t}^{i}(s) d x d \varepsilon d s+t \int_{0}^{t} \int_{\Omega\left(s, \varepsilon_{1}\right)} u_{t}^{i}(s) u_{t}^{i}(s) d x d s \\
& \quad \leq t \int_{0}^{t} \int_{\varepsilon_{1}}^{\varepsilon_{2}} \int_{\partial \Omega(s, \varepsilon)} u_{t}^{i}(s) u_{t}^{i}(s) d x d \varepsilon d s+\frac{2 t}{\varepsilon_{2}-\varepsilon_{1}} \int_{0}^{t} \int_{\varepsilon_{1}}^{\varepsilon_{2}} \mathscr{E}(s, \varepsilon) d \varepsilon d s,
\end{aligned}
$$

for all $0 \leq t \leq t_{0}$. We have used the fact that $\mathscr{E}(s, \varepsilon)$ is nondecreasing in $\varepsilon$. By combining (2.52) and (2.54), we obtain

$$
\int_{\Omega(t, \varepsilon)} u_{\alpha}^{i}(t) u_{\alpha}^{i}(t) d x \leq \frac{2}{d_{1}} \mathscr{E}(t, \varepsilon)+\frac{2 d_{2} t}{d_{1}} \int_{0}^{t} \mathscr{E}(s, \varepsilon) d s,
$$

for all $0 \leq t \leq t_{0}$ and $\varepsilon_{1} \leq \varepsilon \leq \varepsilon_{2}$. It also follows that

$$
\begin{aligned}
\int_{\varepsilon_{1}}^{\varepsilon_{2}} \int_{\partial \Omega(t, \varepsilon)} u_{\alpha}^{i}(t) u_{\alpha}^{i}(t) d x d \varepsilon \leq & \frac{1}{d_{1}} \int_{\varepsilon_{1}}^{\varepsilon_{2}} \int_{\partial \Omega(t, \varepsilon)} C_{i j}^{\alpha \beta} u_{\alpha}^{i}(t) u_{\beta}^{j}(t) d x d \varepsilon \\
& +\frac{d_{2}}{d_{1}} t \int_{0}^{t} \int_{\varepsilon_{1}}^{\varepsilon_{2}} \int_{\partial \Omega(s, \varepsilon)} u_{t}^{i}(s) u_{t}^{i}(s) d x d \varepsilon d s \\
& +\frac{d_{2}}{d_{1}} \frac{2 t}{\varepsilon_{2}-\varepsilon_{1}} \int_{0}^{t} \int_{\varepsilon_{1}}^{\varepsilon_{2}} \mathscr{E}(s, \varepsilon) d \varepsilon d s,
\end{aligned}
$$

for all $0 \leq t \leq t_{0}$.

We now choose $\gamma>0$ such that

$$
\gamma<\min \left(\frac{\eta}{4}, \frac{d_{1}}{4}, \frac{\eta d_{1}}{4}, \frac{\eta d_{1}}{2 d_{2} h^{2}}\right) .
$$

Finally we integrate $(2.48)$ in $\varepsilon$ over $\left[\varepsilon_{1}, \varepsilon_{2}\right]$, and take into account (2.49) through (2.58), so that, for some positive constant $d_{3}$,

$$
\int_{\varepsilon_{1}}^{\varepsilon_{2}} \mathscr{E}(h, \varepsilon) d \varepsilon \leq d_{3} \int_{0}^{h} \int_{\varepsilon_{1}}^{\varepsilon_{2}} \mathscr{E}(t, \varepsilon) d \varepsilon d t
$$

for each $h \in\left[0, t_{0}\right]$. This yields

$$
\mathscr{E}\left(t, \varepsilon_{2}\right)=0, \quad \text { for all } 0 \leq t \leq t_{0} .
$$

We have established the above theorem for $\left(u_{0}, u_{1}\right) \in C_{0}^{\infty}\left(R^{n}\right) \times C_{0}^{\infty}\left(R^{n}\right)$. For $\left(u_{0}, u_{1}\right) \in H^{\nu}\left(R^{n}\right) \times H^{\nu-1}\left(R^{n}\right), \nu \in R$, we start by finding a sequence $\left\{\left(u_{0}^{k}, u_{1}^{k}\right)\right\}_{k=1}^{\infty}$ in $C_{0}^{\infty}\left(R^{n}\right) \times C_{0}^{\infty}\left(R^{n}\right)$ which converges to $\left(u_{0}, u_{1}\right)$ in $H^{\nu}\left(R^{n}\right) \times$ $H^{\nu-1}\left(R^{n}\right)$. We can arrange that if $\Omega(0, \varepsilon)$ is disjoint from supp $u_{0} \cup \operatorname{supp} u_{1}$ for some $\varepsilon>0$, then it is also disjoint from $\operatorname{supp} u_{0}^{k} \cup \operatorname{supp} u_{1}^{k}$. Now we apply the above theorem to the solution with the initial data $\left(u_{0}^{k}, u_{1}^{k}\right)$ for each $k$. Then, we arrive at the conclusion for the true solution $u$ by continuous dependence of solutions on the initial data.

\section{Preliminaries for generalization of MacCamy's trick}

In this section, we develop necessary tools for the proof of Theorem 1.5. First we recall some facts on the basic calculus of pseudodifferential operators. 
Lemma 3.1. $\mathscr{S}^{m}\left([0, T] \times[0, T] \times R^{n} \times R^{n}\right)$ is a Fréchet space with the topology induced by the family of seminorms indexed by $(\alpha, \beta, \gamma, \delta) \in Z_{+} \times Z_{+} \times Z_{+}^{n} \times$ $Z_{+}^{n}$ :

$$
\begin{aligned}
& \sup \left\{(1+|\xi|)^{-m+|\delta|}\left|\partial_{t}^{\alpha} \partial_{s}^{\beta} \partial_{x}^{\gamma} \partial_{\xi}^{\delta} a(t, s, x, \xi)\right|:\right. \\
&\left.(t, s, x, \xi) \in[0, T] \times[0, T] \times R^{n} \times R^{n}\right\}
\end{aligned}
$$

Lemma 3.2. The product map defined by

$$
\left(a_{1}(t, s, x, \xi), a_{2}(t, s, x, \xi)\right) \mapsto a_{1}(t, s, x, \xi) a_{2}(t, s, x, \xi)
$$

is continuous from $\mathscr{S}^{m_{1}}\left([0, T] \times[0, T] \times R^{n} \times R^{n}\right) \times \mathscr{S}^{m_{2}}([0, T] \times[0, T] \times$ $\left.R^{n} \times R^{n}\right)$ into $\mathscr{S}^{m_{1}+m_{2}}\left([0, T] \times[0, T] \times R^{n} \times R^{n}\right)$.

Lemma 3.3. Let $a_{j}(t, s, x, \xi) \in \mathscr{S}^{m_{j}}\left([0, T] \times[0, T] \times R^{n} \times R^{n}\right), j=1,2, \ldots$, be such that $m_{j} \downarrow-\infty$ as $j \rightarrow \infty$. Then, there is

$$
a \in \mathscr{S}^{m_{1}}\left([0, T] \times[0, T] \times R^{n} \times R^{n}\right)
$$

such that

$$
a \sim \sum_{j=1}^{\infty} a_{j}
$$

in a sense that

$$
a-\sum_{j=1}^{N} a_{j} \in \mathscr{S}^{m_{N+1}}\left([0, T] \times[0, T] \times R^{n} \times R^{n}\right),
$$

for every $N \geq 1$.

This can be proved by slightly modifying the proof of Proposition 18.1.3 of [9].

Lemma 3.4. Let $\mathbf{F}_{i}$ be a Fréchet space for $i=1,2,3$. If $\Lambda_{1}$ is a continuous linear mapping from $\mathbf{F}_{1}$ into $\mathbf{F}_{2}$ and $\phi \in C^{\infty}\left([0, T] \times[0, T] ; \mathbf{F}_{1}\right)$, then

$$
\Lambda_{1} \phi \in C^{\infty}\left([0, T] \times[0, T] ; \mathbf{F}_{2}\right) \text {. }
$$

If $\Lambda_{2}$ is a continuous bilinear mapping from $\mathbf{F}_{1} \times \mathbf{F}_{2}$ into $\mathbf{F}_{3}$ and $\psi_{i} \in$ $C^{\infty}\left([0, T] \times[0, T] ; \mathbf{F}_{i}\right), i=1,2$, then

$$
\Lambda_{2}\left(\psi_{1}, \psi_{2}\right) \in C^{\infty}\left([0, T] \times[0, T] ; \mathbf{F}_{3}\right) .
$$

Next, for Banach spaces $\mathbf{B}_{1}$ and $\mathbf{B}_{2}$, we denote by $\mathscr{L}\left(\mathbf{B}_{1} ; \mathbf{B}_{2}\right)$ the Banach space of all continuous linear operators from $\mathbf{B}_{1}$ into $\mathbf{B}_{2}$.

Lemma 3.5. Let $a(t, s, x, \xi) \in \mathscr{S}^{m}\left([0, T] \times[0, T] \times R^{n} \times R^{n}\right)$ for some $m \in R$. Then,

$$
a\left(t, s, x, D_{x}\right) \in C^{\infty}\left([0, T] \times[0, T] ; \mathscr{L}\left(H^{k}\left(R^{n}\right) ; H^{k-m}\left(R^{n}\right)\right)\right),
$$

for each $k \in R$.

Proof. This follows from Lemma 3.4 and the fact that the linear mapping from $\mathscr{S}^{m}\left(R^{n} \times R^{n}\right)$ into $\mathscr{L}\left(H^{k}\left(R^{n}\right) ; H^{k-m}\left(R^{n}\right)\right)$ defined by

$$
b(x, \xi) \mapsto b\left(x, D_{x}\right)
$$

is continuous for each $k \in R$. 
Lemma 3.6. If $A_{i}(t, s, x, \xi)$ is an $N \times N$ matrix and

$$
A_{i}\left(t, s, x, D_{x}\right) \in O P S^{m_{i}}\left([0, T] \times[0, T] \times R^{n}\right),
$$

$m_{i} \in R$, for $i=1,2$, then the composite operator

$$
B=A_{1}\left(t, s, x, D_{x}\right) A_{2}\left(t, s, x, D_{x}\right)
$$

belongs to $O P S^{m_{1}+m_{2}}\left([0, T] \times[0, T] \times R^{n}\right)$. Furthermore, the mapping

$$
\left(A_{1}(t, s, x, \xi), A_{2}(t, s, x, \xi)\right) \mapsto B(t, s, x, \xi)
$$

is continuous from $\mathscr{S}^{m_{1}}\left([0, T] \times[0, T] \times R^{n} \times R^{n}\right) \times \mathscr{S}^{m_{2}}([0, T] \times[0, T] \times$ $\left.R^{n} \times R^{n}\right)$ into $\mathscr{S}^{m_{1}+m_{2}}\left([0, T] \times[0, T] \times R^{n} \times R^{n}\right)$, and

$$
\begin{gathered}
B(t, s, x, \xi)-A_{1}(t, s, x, \xi) A_{2}(t, s, x, \xi) \\
\quad \in \mathscr{S}^{m_{1}+m_{2}-1}\left([0, T] \times[0, T] \times R^{n} \times R^{n}\right) .
\end{gathered}
$$

Proof. This follows from Lemma 3.4 above and Theorem 18.1.8 of [9], which says that the above mapping defined by (3.9) is continuous from $\mathscr{S}^{m_{1}}\left(R^{n} \times R^{n}\right)$ $\times \mathscr{S}^{m_{2}}\left(R^{n} \times R^{n}\right)$ to $\mathscr{S}^{m_{1}+m_{2}}\left(R^{n} \times R^{n}\right)$ and the mapping from the left-hand side of (3.9) to (3.10) is continuous from $\mathscr{S}^{m_{1}}\left(R^{n} \times R^{n}\right) \times \mathscr{S}^{m_{2}}\left(R^{n} \times R^{n}\right)$ to $\mathscr{S}^{m_{1}+m_{2}-1}\left(R^{n} \times R^{n}\right)$.

Lemma 3.7. Let $A(t, x, \xi)=A_{1}(t, x, \xi)+A_{2}(t, x, \xi)$, where $A_{1}(t, x, \xi) \in$ $\mathscr{S}^{m-1}\left([0, T] \times R^{n} \times R^{n}\right)$ and $A_{2}(t, x, \xi) \in \mathscr{S}^{m}\left([0, T] \times R^{n} \times R^{n}\right), m \in R$, are $N \times N$ matrices such that for some positive constant $\eta$,

$$
\left|\operatorname{det} A_{2}(t, x, \xi)\right| \geq \eta \quad \text { if }(t, x) \in[0, T] \times R^{n} \text { and }|\xi|=1 \text {, }
$$

and

$$
A_{2}(t, x, \xi) \text { is homogeneous of degree } m \text { in } \xi \text { for }|\xi| \geq 1 \text {. }
$$

Then, there is an $N \times N$ matrix $B(t, x, \xi) \in \mathscr{S}^{-m}\left([0, T] \times R^{n} \times R^{n}\right)$ such that

$$
\begin{aligned}
& A\left(t, x, D_{x}\right) B\left(t, x, D_{x}\right)-I \in O P S^{-\infty}\left([0, T] \times R^{n}\right), \\
& B\left(t, x, D_{x}\right) A\left(t, x, D_{x}\right)-I \in O P S^{-\infty}\left([0, T] \times R^{n}\right) .
\end{aligned}
$$

Proof. There is a positive constant $c$ such that $A(t, x, \xi)$ is invertible for $|\xi| \geq c$. By virtue of (3.12), we can choose an $N \times N$ matrix $E(t, x, \xi) \in$ $\mathscr{S}^{-m}\left([0, T] \times R^{n} \times R^{n}\right)$ such that

$$
E(t, x, \xi)=A(t, x, \xi)^{-1},
$$

for $|\xi| \geq c$. We then set

$$
R\left(t, x, D_{x}\right)=I-A\left(t, x, D_{x}\right) E\left(t, x, D_{x}\right) .
$$

It follows from (3.10) that

$$
R\left(t, x, D_{x}\right) \in O P S^{-1}\left([0, T] \times R^{n}\right) .
$$

By Lemma 3.3, there is a matrix operator $Q\left(t, x, D_{x}\right) \in O P S^{0}\left([0, T] \times R^{n}\right)$ such that

$$
Q \sim I+R+R^{2}+\cdots
$$

Now let us set

$$
B\left(t, x, D_{x}\right)=E\left(t, x, D_{x}\right) Q\left(t, x, D_{x}\right) .
$$


We claim that this $B$ satisfies (3.13) and (3.14). For this, we set

$$
Q_{m}=I+R+\cdots+R^{m} \text {. }
$$

Then, $I-A E Q_{m}=R^{m+1} \in O P S^{-(m+1)}\left([0, T] \times R^{n}\right)$, which implies that

$$
I-A B \in O P S^{-\infty}\left([0, T] \times R^{n}\right) .
$$

Next, we define

$$
\begin{aligned}
\widetilde{R}\left(t, x, D_{x}\right) & =I-E\left(t, x, D_{x}\right) A\left(t, x, D_{x}\right), \\
\widetilde{Q} & \sim I+\widetilde{R}+\widetilde{R}^{2}+\cdots,
\end{aligned}
$$

and

$$
\widetilde{B}\left(t, x, D_{x}\right)=\widetilde{Q}\left(t, x, D_{x}\right) E\left(t, x, D_{x}\right) .
$$

Then, it follows that

$$
I-\widetilde{B} A \in O P S^{-\infty}\left([0, T] \times R^{n}\right) .
$$

Meanwhile, we obtain from (3.21)

$$
\widetilde{B} A-\widetilde{B} A B A \in O P S^{-\infty}\left([0, T] \times R^{n}\right),
$$

which, combined with (3.25), yields

$$
\widetilde{B} A-\widetilde{B} A B A=I-B A \bmod O P S^{-\infty}\left([0, T] \times R^{n}\right),
$$

and hence, (3.14) follows.

For generalization of MacCamy's trick, we shall use a particular version of Beals's result [1] on the characterization of pseudodifferential operators. First of all, let us introduce some necessary notation:

$$
\begin{gathered}
\mathscr{S}\left(R^{n}\right)=\text { the space of } C^{\infty} \text { functions in } R^{n} \\
\text { which decay rapidly at infinity, } \\
\mathscr{S}^{\prime}\left(R^{n}\right)=\text { the dual of } \mathscr{S}\left(R^{n}\right) .
\end{gathered}
$$

As above, if $f$ is $\mathscr{C}^{N}$ valued and each component of $f$ belongs to $\mathscr{S}\left(R^{n}\right)$ (resp. $\mathscr{S}^{\prime}\left(R^{n}\right)$ ), we still write $f \in \mathscr{S}\left(R^{n}\right)$ (resp. $\mathscr{S}^{\prime}\left(R^{n}\right)$ ). For a linear operator $B$ from $\mathscr{S}\left(R^{n}\right)$ into $\mathscr{S}^{\prime}\left(R^{n}\right)$, we define

$$
\begin{gathered}
-L_{j} B=\left[\sqrt{-1} x_{j}, B\right]=\sqrt{-1} x_{j} B-\sqrt{-1} B x_{j}, \\
M_{j} B=\left[D_{j}, B\right]=D_{j} B-B D_{j},
\end{gathered}
$$

for $j=1, \ldots, n$, and

$$
\begin{gathered}
L^{\gamma}=L_{1}^{\gamma_{1}} \cdots L_{n}^{\gamma_{n}}, \quad \text { for } \gamma=\left(\gamma_{1}, \ldots, \gamma_{n}\right) \in Z_{+}^{n}, \\
M^{\delta}=M_{1}^{\delta_{1}} \cdots M_{n}^{\delta_{n}}, \quad \text { for } \delta=\left(\delta_{1}, \ldots, \delta_{n}\right) \in Z_{+}^{n} .
\end{gathered}
$$

Next we define a set of $N \times N$ matrix operators:

$$
\mathscr{X}=\left\{B: B \text { is a linear operator from } \mathscr{S}\left(R^{n}\right) \text { to } \mathscr{S}^{\prime}\left(R^{n}\right)\right. \text { and satisfies }
$$$$
\left.L^{\gamma} M^{\delta} B \in \mathscr{L}\left(H^{-|\gamma|}\left(R^{n}\right) ; L^{2}\left(R^{n}\right)\right) \text {, for every }(\gamma, \delta) \in Z_{+}^{n} \times Z_{+}^{n}\right\} \text {, }
$$ 
which is equipped with the topology induced by the family of seminorms:

$$
\left\|L^{\gamma} M^{\delta} B\right\|_{\gamma}, \quad \text { for }(\gamma, \delta) \in Z_{+}^{n} \times Z_{+}^{n} \text {. }
$$

Here $\|\cdot\|_{\gamma}$ is the operator norm from $H^{-|\gamma|}\left(R^{n}\right)$ into $L^{2}\left(R^{n}\right)$. The following is a particular matrix version of Theorem 2.9 of [1].

Lemma 3.8. If $B \in \mathscr{X}$, then $L^{\gamma} M^{\delta} B \in O P S^{-|\gamma|}\left(R^{n}\right)$ for each $(\gamma, \delta) \in Z_{+}^{n} \times Z_{+}^{n}$, and the linear map $B \mapsto b(x, \xi)$ is a topological isomorphism from $\mathscr{X}$ onto $\mathscr{S}^{0}\left(R^{n} \times R^{n}\right)$, where $B=b\left(x, D_{x}\right)$.

Combining this with Lemma 3.4, we infer

Lemma 3.9. Let $B(t, s)$ be an $N \times N$ matrix operator such that $B(t, s)$ is a linear operator from $\mathscr{S}\left(R^{n}\right)$ to $\mathscr{S}^{\prime}\left(R^{n}\right)$ for each $(t, s) \in[0, T] \times[0, T]$. Then, we have

(i) If $L^{\gamma} M^{\delta} B(t, s) \in C^{\infty}\left([0, T] \times[0, T] ; \mathscr{L}\left(H^{-|\gamma|}\left(R^{n}\right) ; L^{2}\left(R^{n}\right)\right)\right)$ for each $(\gamma, \delta) \in Z_{+}^{n} \times Z_{+}^{n}$, then $B(t, s) \in O P S^{0}\left([0, T] \times[0, T] \times R^{n}\right)$.

(ii) If $B(t, s) \in O P S^{0}\left([0, T] \times[0, T] \times R^{n}\right)$, then for each $\rho \in R$,

$$
L^{\gamma} M^{\delta} B(t, s) \in C^{\infty}\left([0, T] \times[0, T] ; \mathscr{L}\left(H^{-|\gamma|+\rho}\left(R^{n}\right) ; H^{\rho}\left(R^{n}\right)\right)\right) .
$$

Now we proceed to prove the following fact.

Theorem 3.10. Let $A$ be an $N \times N$ matrix operator such that $A\left(t, s, x, D_{x}\right) \in$ $O P S^{0}\left([0, T] \times[0, T] \times R^{n}\right)$. Define $A_{1}=A$ and

$$
A_{k+1}\left(t, s, x, D_{x}\right)=\int_{s}^{t} A_{1}\left(t, \eta, x, D_{x}\right) A_{k}\left(\eta, s, x, D_{x}\right) d \eta,
$$

for $k \geq 1$. Then, for each $m \geq 1$,

$$
B_{m}\left(t, s, x, D_{x}\right)=\sum_{k=1}^{m} A_{k}\left(t, s, x, D_{x}\right) \in O P S^{0}\left([0, T] \times[0, T] \times R^{n}\right)
$$

and, as $m \rightarrow \infty$,

$$
B_{m} \rightarrow B \quad \text { in } C^{\infty}([0, T] \times[0, T] ; \mathscr{X}),
$$

for some $B\left(t, s, x, D_{x}\right) \in O P S^{0}\left([0, T] \times[0, T] \times R^{n}\right)$.

For the proof of this theorem, we need some preparation.

Lemma 3.11. For any $m=m_{1}+m_{2}, m_{1} \geq 1, m_{2} \geq 1$,

$$
A_{m}\left(t, s, x, D_{x}\right)=\int_{s}^{t} A_{m_{1}}\left(t, \eta, x, D_{x}\right) A_{m_{2}}\left(\eta, s, x, D_{x}\right) d \eta .
$$

We can easily derive (3.39) by induction.

Lemma 3.12. If $m \geq m_{1}+m_{2}+2$, then

$$
\left.\partial_{t}^{m_{1}} \partial_{s}^{m_{2}} A_{m}\left(t, s, x, D_{x}\right)\right|_{s=t}=0
$$

in $\operatorname{OPS}^{0}\left([0, T] \times R^{n}\right)$.

Proof. (3.40) is obvious for $m=2, m_{1}=m_{2}=0$. Assume that (3.40) is true for $2 \leq m \leq r, m \geq m_{1}+m_{2}+2$. For any $m_{1} \geq 0, m_{2} \geq 0, m_{1}+m_{2} \geq 1$, 
we have

(3.41)

$$
\begin{aligned}
\partial_{t}^{m_{1}} \partial_{s}^{m_{2}} & A_{r+1}\left(t, s, x, D_{x}\right) \\
= & \left.\sum C_{1}\left(k_{1}, k_{2}, l_{1}, l_{2}\right) \partial_{t}^{k_{1}} \partial_{\eta}^{l_{1}} A_{1}\left(t, \eta, x, D_{x}\right) \partial_{\eta}^{k_{2}} \partial_{s}^{l_{2}} A_{r}\left(\eta, s, x, D_{x}\right)\right|_{\eta=t} \\
& +\left.\sum C_{2}\left(k_{1}, k_{2}, l_{1}, l_{2}\right) \partial_{t}^{k_{1}} \partial_{\eta}^{l_{1}} A_{1}\left(t, \eta, x, D_{x}\right) \partial_{\eta}^{k_{2}} \partial_{s}^{l_{2}} A_{r}\left(\eta, s, x, D_{x}\right)\right|_{\eta=s} \\
& +\int_{s}^{t} \partial_{t}^{m_{1}} A_{1}\left(t, \eta, x, D_{x}\right) \partial_{s}^{m_{2}} A_{r}\left(\eta, s, x, D_{x}\right) d \eta
\end{aligned}
$$

where $C_{1}$ and $C_{2}$ are nonnegative constants depending on $k_{1}, k_{2}, l_{1}$ and $l_{2}$, and the summation is taken over

$$
k_{1}+k_{2}+l_{1}+l_{2} \leq m_{1}+m_{2}-1 .
$$

Hence, if $m_{1}+m_{2}+2 \leq r+1$, then $k_{2}+l_{2}+2 \leq m_{1}+m_{2}+1 \leq r$ and thus,

$$
\left.\partial_{\eta}^{k_{2}} \partial_{s}^{l_{2}} A_{r}\left(\eta, s, x, D_{x}\right)\right|_{\eta=s}=0 \text {. }
$$

Therefore, if $m_{1}+m_{2}+2 \leq r+1$, then

$$
\begin{aligned}
\partial_{t}^{m_{1}} \partial_{s}^{m_{2}} & A_{r+1}\left(t, s, x, D_{x}\right) \\
= & \left.\sum C_{1}\left(k_{1}, k_{2}, l_{1}, l_{2}\right) \partial_{t}^{k_{1}} \partial_{\eta}^{l_{1}} A_{1}\left(t, \eta, x, D_{x}\right)\right|_{\eta=t} \partial_{t}^{k_{2}} \partial_{s}^{l_{2}} A_{r}\left(t, s, x, D_{x}\right) \\
& +\int_{s}^{t} \partial_{t}^{m_{1}} A_{1}\left(t, \eta, x, D_{x}\right) \partial_{s}^{m_{2}} A_{r}\left(\eta, s, x, D_{x}\right) d \eta .
\end{aligned}
$$

As above, if $m_{1}+m_{2}+2 \leq r+1$, then

$$
\left.\partial_{t}^{k_{2}} \partial_{s}^{l_{2}} A_{r}\left(t, s, x, D_{x}\right)\right|_{t=s}=0 .
$$

Consequently, we have

$$
\left.\partial_{t}^{m_{1}} \partial_{s}^{m_{2}} A_{r+1}\left(t, s, x, D_{x}\right)\right|_{t=s}=0,
$$

if $m_{1}+m_{2}+2 \leq r+1$. By induction, (3.40) is valid for all $m \geq 2$.

The following formula of expansion is also useful later on.

Lemma 3.13. Let $\Lambda_{1}$ and $\Lambda_{2}$ be linear operators from $\mathscr{S}\left(R^{n}\right)$ into itself. Then, for each $(\alpha, \beta) \in Z_{+}^{n} \times Z_{+}^{n}$,

$$
L^{\alpha} M^{\beta} \Lambda_{1} \Lambda_{2}=\sum_{\gamma \leq \alpha} \sum_{\delta \leq \beta}\left(\begin{array}{l}
\alpha \\
\gamma
\end{array}\right)\left(\begin{array}{l}
\beta \\
\delta
\end{array}\right)\left(L^{\gamma} M^{\delta} \Lambda_{1}\right)\left(L^{\alpha-\gamma} M^{\beta-\delta} \Lambda_{2}\right) .
$$

Proof of Theorem 3.10. Fix any $n_{j} \in Z_{+}, j=1,2,3,4$, and set $\sigma=n_{1}+$ $n_{2}+2$. We shall show that

$$
\left\|L^{\alpha} M^{\beta} \partial_{t}^{m_{1}} \partial_{s}^{m_{s}} A_{2 k \sigma}\left(t, s, x, D_{x}\right)\right\|_{\alpha} \leq C^{k}|t-s|^{k} / k !,
$$

for all $(t, s) \in[0, T] \times[0, T], k \geq 1, m_{1} \leq n_{1}, m_{2} \leq n_{2}$, and $(\alpha, \beta) \in$ $Z_{+}^{n} \times Z_{+}^{n}$ satisfying $|\alpha| \leq n_{3}$ and $|\beta| \leq n_{4}$, where $C$ depends on $n_{1}, n_{2}$, $n_{3}, n_{4}$, but is independent of $k$. Here $\|\cdot\|_{\alpha}$ denotes the operator norm from 
$H^{-|\alpha|}\left(R^{n}\right)$ into $L^{2}\left(R^{n}\right)$. When $k=1, m_{1} \leq n_{1}$ and $m_{2} \leq n_{2}$, we use Lemmas $3.11,3.12$ and 3.13 to derive

$$
\begin{aligned}
& L^{\alpha} M^{\beta} \partial_{t}^{m_{1}} \partial_{s}^{m_{2}} A_{2 \sigma}\left(t, s, x, D_{x}\right) \\
&=L^{\alpha} M^{\beta} \int_{s}^{t} \partial_{t}^{m_{1}} A_{\sigma}\left(t, \eta, x, D_{x}\right) \partial_{s}^{m_{2}} A_{\sigma}\left(\eta, s, x, D_{x}\right) d \eta \\
&=\sum_{\gamma \leq \alpha} \sum_{\delta \leq \beta}\left(\begin{array}{l}
\alpha \\
\gamma
\end{array}\right)\left(\begin{array}{c}
\beta \\
\delta
\end{array}\right) \int_{s}^{t}\left\{L^{\gamma} M^{\delta} \partial_{t}^{m_{1}} A_{\sigma}\left(t, \eta, x, D_{x}\right)\right\} \\
& \quad \times\left\{L^{\alpha-\gamma} M^{\beta-\delta} \partial_{s}^{m_{2}} A_{\sigma}\left(\eta, s, x, D_{x}\right)\right\} d \eta .
\end{aligned}
$$

According to Lemma 3.9, there is a positive constant $d_{1}$ depending on $n_{1}, n_{2}$, $n_{3}$ and $n_{4}$ such that

$$
\left\|L^{\alpha-\gamma} M^{\beta-\delta} \partial_{s}^{m_{2}} A_{\sigma}\left(\eta, s, x, D_{x}\right)\right\|_{\alpha, \gamma} \leq d_{1},
$$

and

$$
\left\|L^{\gamma} M^{\delta} \partial_{t}^{m_{1}} A_{\sigma}\left(t, \eta, x, D_{x}\right)\right\|_{\gamma} \leq d_{1},
$$

for all $(\eta, s),(t, \eta) \in[0, T] \times[0, T]$ and all $\alpha, \beta, \gamma, \delta, m_{1}$ and $m_{2}$ satisfying $|\alpha| \leq n_{3},|\beta| \leq n_{4}, \gamma \leq \alpha, \delta \leq \beta, m_{1} \leq n_{1}$ and $m_{2} \leq n_{2}$. Here $\|\cdot\|_{\alpha, \gamma}$ denotes the operator norm from $H^{-|\alpha|}\left(R^{n}\right)$ into $H^{-|\gamma|}\left(R^{n}\right)$ and $\|\cdot\|_{\gamma}$ denotes the operator norm from $H^{-|\gamma|}\left(R^{n}\right)$ into $L^{2}\left(R^{n}\right)$. Now we use

$$
\sum_{\gamma \leq \alpha} \sum_{\delta \leq \beta}\left(\begin{array}{l}
\alpha \\
\gamma
\end{array}\right)\left(\begin{array}{l}
\beta \\
\delta
\end{array}\right) \leq 2^{n_{3}+n_{4}}
$$

to derive (3.48) with $C=C_{1} \stackrel{\text { def }}{=} 2^{n_{3}+n_{4}} d_{1}^{2}$. Next let us suppose that (3.48) is valid for $1 \leq k \leq q$, for some $C \geq C_{1}$. Then, again by Lemmas 3.11, 3.12 and 3.13, we have

$$
\begin{aligned}
L^{\alpha} M^{\beta} \partial_{t}^{m_{1}} \partial_{s}^{m_{2}} A_{2(q+1) \sigma}\left(t, s, x, D_{x}\right) & \\
= & L^{\alpha} M^{\beta} \partial_{t}^{m_{1}} \partial_{s}^{m_{2}} \int_{s}^{t} A_{2 q \sigma}\left(t, \eta, x, D_{x}\right) A_{2 \sigma}\left(\eta, s, x, D_{x}\right) d \eta \\
=\sum_{\gamma \leq \alpha} \sum_{\delta \leq \beta}\left(\begin{array}{l}
\alpha \\
\gamma
\end{array}\right)\left(\begin{array}{c}
\beta \\
\delta
\end{array}\right) \int_{s}^{t} & \left\{L^{\gamma} M^{\delta} \partial_{t}^{m_{1}} A_{2 q \sigma}\left(t, \eta, x, D_{x}\right)\right\} \\
& \times\left\{L^{\alpha-\gamma} M^{\beta-\delta} \partial_{s}^{m_{2}} A_{2 \sigma}\left(\eta, s, x, D_{x}\right)\right\} d \eta,
\end{aligned}
$$

for $m_{1} \leq n_{1}$ and $m_{2} \leq n_{2}$. By means of (3.48) for $k=q$, we obtain

$$
\left\|L^{\gamma} M^{\delta} \partial_{t}^{m_{1}} A_{2 q \sigma}\left(t, \eta, x, D_{x}\right)\right\|_{\gamma} \leq C^{q}|t-\eta|^{q} / q !,
$$

for all $(t, \eta) \in[0, T] \times[0, T]$ and all $\gamma, \delta, m_{1}$ satisfying $|\gamma| \leq n_{3},|\delta| \leq n_{4}$ and $m_{1} \leq n_{1}$. Meanwhile, by the same argument as for (3.50), we find that there is a positive constant $C_{2}$ depending only on $n_{1}, n_{2}, n_{3}$, and $n_{4}$ such that

$$
\left\|L^{\alpha-\gamma} M^{\beta-\delta} \partial_{s}^{m_{2}} A_{2 \sigma}\left(\eta, s, x, D_{x}\right)\right\|_{\alpha, \gamma} \leq C_{2},
$$

for all $(\eta, s) \in[0, T] \times[0, T]$ and all $\alpha, \beta, \gamma, \delta, m_{2}$ satisfying $\gamma \leq \alpha$, $\delta \leq \beta,|\alpha| \leq n_{3},|\beta| \leq n_{4}$ and $m_{2} \leq n_{2}$. Combining (3.52) through (3.55), we obtain

$$
\begin{gathered}
\left\|L^{\alpha} M^{\beta} \partial_{t}^{m_{1}} \partial_{s}^{m_{2}} A_{2(q+1) \sigma}\left(t, s, x, D_{x}\right)\right\|_{\alpha} \\
\leq 2^{n_{3}+n_{4}} C_{2} C^{q}|t-s|^{q+1} /(q+1) ! .
\end{gathered}
$$


Hence, if we take

$$
C=\max \left\{C_{1}, 2^{n_{3}+n_{4}} C_{2}\right\},
$$

(3.48) is valid for all $k \geq 1$. Next we fix any $1 \leq \mu \leq 2 \sigma-1$. By the same argument as above, we have

$$
\left\|L^{\alpha} M^{\beta} \partial_{t}^{m_{1}} \partial_{s}^{m_{2}} A_{2 k \sigma+\mu}\left(t, s, x, D_{x}\right)\right\|_{\alpha} \leq C^{k}|t-s|^{k} / k !,
$$

for all $(t, s) \in[0, T] \times[0, T], k \geq 1, m_{1} \leq n_{1}, m_{2} \leq n_{2}$ and $(\alpha, \beta) \in$ $Z_{+}^{n} \times Z_{+}^{n}$ satisfying $|\alpha| \leq n_{3}$ and $|\beta| \leq n_{4}$, where $C$ depends on $n_{1}, n_{2}, n_{3}$, $n_{4}$, but is independent of $k$. With help of (3.48) and (3.58), we apply Lemma 3.9 to $B$ and conclude the proof.

A crucial step in MacCamy's trick is to represent solutions of a Volterra integral equation. Here we deal with a Volterra integral equation whose kernel is a matrix pseudodifferential operator. Let $A\left(t, s, x, D_{x}\right)$ be an $N \times N$ matrix operator which belongs to $\operatorname{OPS}^{0}\left([0, T] \times[0, T] \times R^{n} \times R^{n}\right)$, and consider a Volterra integral equation

$$
v(t)=u(t)-\int_{0}^{t} A\left(t, s, x, D_{x}\right) u(s) d s,
$$

for $0 \leq t \leq T$, where $u(t)$ and $v(t)$ are $\mathscr{C}^{N}$ valued and belong to $H^{\mu}\left(R^{n}\right)$ for some $\mu \in R$.

Theorem 3.14. For given $v(t) \in C\left([0, T] ; H^{\mu}\left(R^{n}\right)\right), \mu \in R$, the solution $u(t)$ of (3.59) can be represented by

$$
u(t)=v(t)+\int_{0}^{t} B\left(t, s, x, D_{x}\right) v(s) d s,
$$

where $B\left(t, s, x, D_{x}\right)$ is an $N \times N$ matrix operator which belongs to the space $O P S^{0}\left([0, T] \times[0, T] \times R^{n}\right)$.

Proof. Let us define

$$
B_{m}\left(t, s, x, D_{x}\right)=\sum_{k=1}^{m} A_{k}\left(t, s, x, D_{x}\right),
$$

where we write $A_{1}=A$ and

$$
A_{k+1}\left(t, s, x, D_{x}\right)=\int_{s}^{t} A_{1}\left(t, \eta, x, D_{x}\right) A_{k}\left(\eta, s, x, D_{x}\right) d \eta,
$$

for $k \geq 1$. Next we set

$$
u_{m}(t)=v(t)+\int_{0}^{t} B_{m}\left(t, s, x, D_{x}\right) v(s) d s,
$$

for $m \geq 1$. Then, it follows that

$$
\begin{aligned}
\int_{0}^{t} A\left(t, s, x, D_{x}\right) u_{m}(s) d s \\
=\int_{0}^{t} A\left(t, s, x, D_{x}\right) v(s) d s \\
\quad+\int_{0}^{t} A\left(t, s, x, D_{x}\right)\left\{\int_{0}^{s} B_{m}\left(s, \eta, x, D_{x}\right) v(\eta) d \eta\right\} d s \\
=\int_{0}^{t} B_{m+1}\left(t, s, x, D_{x}\right) v(s) d s .
\end{aligned}
$$


By virtue of Theorem 3.10, there is an operator

$$
B\left(t, s, x, D_{x}\right) \in O P S^{0}\left([0, T] \times[0, T] \times R^{n}\right)
$$

such that

$$
B=\lim _{m \rightarrow \infty} B_{m} \text { in } C^{\infty}([0, T] \times[0, T] ; \mathscr{X}),
$$

from which it follows that

$$
\lim _{m \rightarrow \infty} u_{m}=u \text { in } C\left([0, T] ; H^{\mu}\left(R^{n}\right)\right),
$$

for some $u \in C\left([0, T] ; H^{\mu}\left(R^{n}\right)\right)$. By combining (3.63) through (3.66), we find that

$$
u(t)=v(t)+\int_{0}^{t} B\left(t, s, x, D_{x}\right) v(s) d s
$$

and

$$
\int_{0}^{t} A\left(t, s, x, D_{x}\right) u(s) d s=\int_{0}^{t} B\left(t, s, x, D_{x}\right) v(s) d s .
$$

Consequently, $u$ satisfies (3.59). Finally, the uniqueness of the solution of (3.59) follows from Gronwall's inequality, and the unique solution is represented by (3.60).

\section{Proof of Theorem 1.5}

According to Theorem 2.1, there is a unique solution $u(x, t)$ of $(0.1)$ and (0.2) for given $\left(u_{0}, u_{1}\right) \in H^{\nu}\left(R^{n}\right) \times H^{\nu-1}\left(R^{n}\right)$ such that

$$
u \in C^{k}\left([0, \infty) ; H^{\nu-k}\left(R^{n}\right)\right),
$$

for all $k \in Z_{+}$. (4.1) will be used whenever necessary. We shall proceed according to the general strategy outlined in $\S 1$.

4.1. Reformulation of (0.1). Let us define $n \times n$ matrices $L$ and $\Phi$ in terms of their entries:

$$
\begin{gathered}
L_{i j}(t, x, \xi)=-C_{i j}^{\alpha \beta}(t, x) \xi_{\alpha} \xi_{\beta}+\sqrt{-1} D_{i j}^{\alpha}(t, x) \xi_{\alpha}, \\
\Phi_{i j}(t, s, x, \xi)=-M_{i j}^{\alpha \beta}(t, s, x) \xi_{\alpha} \xi_{\beta}+\sqrt{-1} N_{i j}^{\alpha}(t, s, x) \xi_{\alpha},
\end{gathered}
$$

for $t \in[0, \infty), s \in[0, \infty), x \in R^{n}$ and $\xi \in R^{n}$. Then, (0.1) can be rewritten as

$$
u_{t t}=L\left(t, x, D_{x}\right) u+\int_{0}^{t} \Phi\left(t, s, x, D_{x}\right) u(s) d s .
$$

We fix a positive number $T$ such that

$$
T>t^{*} \text {, }
$$

where $t^{*}$ appeared in Theorem 1.5. The operator $L\left(t, x, D_{x}\right)$ satisfies the conditions in Lemma 3.7. Thus, there is a parametrix

$$
\widetilde{L}\left(t, x, D_{x}\right) \in O P S^{-2}\left([0, T] \times R^{n}\right)
$$


such that

$$
\begin{aligned}
L\left(t, x, D_{x}\right) \widetilde{L}\left(t, x, D_{x}\right) & =\widetilde{L}\left(t, x, D_{x}\right) L\left(t, x, D_{x}\right) \\
& =I \bmod O P S^{-\infty}\left([0, T] \times R^{n}\right),
\end{aligned}
$$

where $I$ is the identity mapping. Next we write

$$
Q\left(t, s, x, D_{x}\right)=\widetilde{L}\left(t, x, D_{x}\right) \Phi\left(t, s, x, D_{x}\right),
$$

so that

$$
Q\left(t, s, x, D_{x}\right) \in O P S^{0}\left([0, T] \times[0, T] \times R^{n}\right),
$$

and define

$$
v(t)=u(t)+\int_{0}^{t} Q\left(t, s, x, D_{x}\right) u(s) d s .
$$

By virtue of Theorem 3.14, there is an $n \times n$ matrix operator $R\left(t, s, x, D_{x}\right) \in$ $O P S^{0}\left([0, T] \times[0, T] \times R^{n}\right)$ such that

$$
u(t)=v(t)+\int_{0}^{t} R\left(t, s, x, D_{x}\right) v(s) d s .
$$

In terms of $v,(4.4)$ is equivalent to

$$
\begin{aligned}
v_{t t}= & L v+R_{1}\left(t, x, D_{x}\right) v_{t}+R_{2}\left(t, x, D_{x}\right) v \\
& -\int_{0}^{t} R_{t t}\left(t, s, x, D_{x}\right) v(s) d s+f
\end{aligned}
$$

where 

$A(t, x, \xi)$ is homogeneous of degree zero in $\xi$ for $|\xi| \geq 1$,

(4.19) $\quad|\operatorname{det} A(t, x, \xi)| \geq c, \quad$ for every $(t, x, \xi)$ satisfying $|\xi| \geq 1$,

$$
A(t, x, \xi)^{-1}\left[C_{i j}^{\alpha \beta}(t, x) \xi_{\alpha} \xi_{\beta}\right] A(t, x, \xi)=\left(\begin{array}{ccc}
J_{1} & & 0 \\
& \ddots & \\
0 & & J_{p}
\end{array}\right),
$$

for all $(t, x, \xi) \in \mathscr{N}$ satisfying $|\xi| \geq 1$, where the last matrix is a block diagonal matrix with

$$
\begin{gathered}
J_{k}=\lambda_{k}(t, x, \xi) I_{k}, \quad k=1, \ldots, p, \\
I_{k}=\text { the } n_{k} \times n_{k} \text { identity matrix, } k=1, \ldots, p .
\end{gathered}
$$

Here $n_{k}$ is the algebraic multiplicity of the eigenvalue $\lambda_{k}$. By (4.18), (4.19) and Lemma 3.7, there is a parametrix $B\left(t, x, D_{x}\right) \in O P S^{0}\left([0, T] \times R^{n}\right)$ such that

$$
\text { the principal symbol of } B \text { is } A(t, x, \xi)^{-1} \text { for }|\xi| \geq 1 \text {, }
$$

$$
\begin{aligned}
A\left(t, x, D_{x}\right) B\left(t, x, D_{x}\right) & =B\left(t, x, D_{x}\right) A\left(t, x, D_{x}\right) \\
& =I \bmod O P S^{-\infty}\left([0, T] \times R^{n}\right) .
\end{aligned}
$$

Next let us fix any $l=1, \ldots, p$, and denote by $\Gamma_{l}(t)=(t, x(t), \tau(t), \xi(t))$ the bicharacteristic strip which corresponds to $\mathscr{P}_{l}(t, x, \tau, \xi)$ defined by $(1.9)$ and satisfies

$$
x\left(t^{*}\right)=x^{*}, \quad \tau\left(t^{*}\right)=\tau^{*}, \quad \xi\left(t^{*}\right)=\xi^{*},
$$

where $t^{*}>0$ is the same as above and $\left(\tau^{*}, \xi^{*}\right) \neq(0,0)$. We restrict to

$$
\gamma_{l}(t)=(t, x(t), \xi(t)) .
$$

Then, there are $t_{m}$, a conic neighborhood $\mathscr{N}_{m}$ of $\gamma_{l}\left(t_{m}\right)$ in $[0, \infty) \times$ $R^{n} \times\left(R^{n} \backslash\{0\}\right)$ and $n \times n$ matrix operators $A_{m}\left(t, x, D_{x}\right), B_{m}\left(t, x, D_{x}\right) \in$ $O P S^{0}\left([0, T] \times R^{n}\right)$, for $m=0,1, \ldots, q$, such that

$$
\begin{gathered}
0=t_{0}<t_{1}<\cdots<t_{q}=t^{*}, \\
\bigcup_{t_{m} \leq t \leq t_{m+1}} \gamma_{l}(t) \subset \mathscr{N}_{m}, \quad m=0,1, \ldots, q-1,
\end{gathered}
$$

(4.29) the principal symbol of $B_{m}\left(t, x, D_{x}\right)$ is $A_{m}(t, x, \xi)^{-1}$ for $|\xi| \geq 1$,

$$
\begin{aligned}
A_{m}\left(t, x, D_{x}\right) B_{m}\left(t, x, D_{x}\right) & =B_{m}\left(t, x, D_{x}\right) A_{m}\left(t, x, D_{x}\right) \\
& =I \bmod O P S^{-\infty}\left([0, T] \times R^{n}\right),
\end{aligned}
$$

$$
A_{m}(t, x, \xi)^{-1}\left[C_{i j}^{\alpha \beta}(t, x) \xi_{\alpha} \xi_{\beta}\right] A_{m}(t, x, \xi) \text { is diagonal as in (4.20), }
$$

for all $(t, x, \xi) \in \mathscr{N}_{m},|\xi| \geq 1$.

Remark 4.1. In general, there is not a global smooth diagonalizing matrix. For (1.35), a global smooth diagonalizer does not exist on account of the hairy ball theorem.

Next we recall the following definition on microlocal regularity. 
Definition 4.2. Let $w \in \mathscr{D}^{\prime}\left(R^{n+1}\right)$ and $\sigma \in R$. We say that $w$ is $H^{\sigma}$ at $\left(t_{0}, x_{0}, \tau_{0}, \xi_{0}\right) \in R^{n+1} \times\left(R^{n+1} \backslash\{0\}\right)$ if $w=w_{1}+w_{2}$ with

$$
\begin{gathered}
w_{1} \in H^{\sigma}\left(R^{n+1}\right), \\
\left(t_{0}, x_{0}, \tau_{0}, \xi_{0}\right) \notin W F\left(w_{2}\right) .
\end{gathered}
$$

We are now prepared to discuss the microlocal regularity of solutions of the equation:

$$
\begin{aligned}
w_{t t}= & L\left(t, x, D_{x}\right) w+F_{1}\left(t, x, D_{x}\right) w_{t} \\
& +F_{2}\left(t, x, D_{x}\right) w+f \text { in }(0, T) \times R^{n},
\end{aligned}
$$

where the symbol of $L$ was defined by (4.2), and $F_{1}$ and $F_{2}$ are $n \times n$ matrix operators such that

$$
\begin{aligned}
& F_{1}\left(t, x, D_{x}\right) \in O P S^{0}\left([0, T] \times R^{n}\right), \\
& F_{2}\left(t, x, D_{x}\right) \in O P S^{1}\left([0, T] \times R^{n}\right) .
\end{aligned}
$$

Using the same notation as above, we fix $l=1, \ldots, p$ and $m=1, \ldots, q-1$, and assert the following.

Lemma 4.3. Let $\mu \leq \sigma-1$ and assume that $w \in C^{k}\left([0, T] ; H^{\mu-k}\left(R^{n}\right)\right), \quad$ for all $k \in Z_{+}$,

$$
w \text { and } f \text { are } H^{\sigma-1} \text { at } \bigcup_{t_{m} \leq t \leq t_{m+1}} \Gamma_{l}(t) \text {, }
$$

$$
w \text { is } H^{\sigma} \text { at } \Gamma_{l}\left(t_{m}\right) \text {. }
$$

Then, $w$ is $H^{\sigma}$ at $\Gamma_{l}(t), t_{m} \leq t \leq t_{m+1}$.

Proof. Let $B_{m}\left(t, x, D_{x}\right)$ be the same as above, and set

$$
y=B_{m}\left(t, x, D_{x}\right) w .
$$

It is evident that

$$
y \in C^{k}\left([0, T] ; H^{\mu-k}\left(R^{n}\right)\right), \quad \text { for all } k \in Z_{+},
$$

and

$$
A_{m}\left(t, x, D_{x}\right) y=w+g,
$$

for some $g \in C^{\infty}\left([0, T] ; H^{k}\left(R^{n}\right)\right)$, for all $k \in Z_{+}$. Consequently, we find that

$$
y_{t t}=B_{m} L A_{m} y+G_{1} y_{t}+G_{2} y+h,
$$

where

$$
\begin{gathered}
G_{1}=B_{m} F_{1} A_{m}-2 B_{m} A_{m t}, \\
G_{2}=-B_{m} A_{m t t}+B_{m} F_{1} A_{m t}+B_{m} F_{2} A_{m}, \\
h=B_{m} f+\left(I-B_{m} A_{m}\right) y_{t t}-B_{m} L g-B_{m} F_{1} g_{t}-B_{m} F_{2} g+B_{m} g_{t t} .
\end{gathered}
$$

On account of (4.35) and (4.36), it is apparent that

$$
\begin{aligned}
& G_{1}\left(t, x, D_{x}\right) \in O P S^{0}\left([0, T] \times R^{n}\right), \\
& G_{2}\left(t, x, D_{x}\right) \in O P S^{1}\left([0, T] \times R^{n}\right) .
\end{aligned}
$$

We need a special version of Theorem 18.1.35 of [9]. 
Lemma 4.4. If $H\left(t, x, D_{x}\right)$ is an $n \times n$ matrix operator in $O P S^{m}\left([0, T] \times R^{n}\right)$, for some $m \in R$, then

$$
\begin{aligned}
& \psi(t) H\left(t, x, D_{x}\right) \Psi\left(t, x, D_{t}, D_{x}\right) \in O P S^{m}\left(R^{n+1}\right), \\
& \Psi\left(t, x, D_{t}, D_{x}\right) \psi(t) H\left(t, x, D_{x}\right) \in O P S^{m}\left(R^{n+1}\right),
\end{aligned}
$$

for any $\psi(t) \in C_{0}^{\infty}((0, T))$ and any $n \times n$ matrix operator $\Psi\left(t, x, D_{t}, D_{x}\right) \in$ $O P S^{0}\left(R^{n+1}\right)$ that satisfies

$$
\Psi(t, x, \tau, \xi)=0,
$$

for all $(t, x, \tau, \xi) \in R^{n+1} \times R^{n+1}$ such that $|\xi| \leq \varepsilon|\tau|$ and $|\tau|>1 / \varepsilon$, for some $\varepsilon>0$.

By virtue of this, $B_{m}\left(t, x, D_{x}\right), G_{1}\left(t, x, D_{x}\right)$ and $G_{2}\left(t, x, D_{x}\right)$ are matrix pseudodifferential operators in $(t, x)$ microlocally along $\Gamma_{l}(t)$. Consequently, we find that

$$
\begin{aligned}
& y \text { is } H^{\sigma-1} \text { at } \Gamma_{l}(t), \quad t_{m} \leq t \leq t_{m+1}, \\
& y \text { is } H^{\sigma} \text { at } \Gamma_{l}\left(t_{m}\right), \\
& h \text { is } H^{\sigma-1} \text { at } \Gamma_{l}(t), \quad t_{m} \leq t \leq t_{m+1} .
\end{aligned}
$$

Meanwhile, for $(t, x, \xi) \in \mathcal{N}_{m}$ satisfying $|\xi| \geq 1$, the principal symbol of $B_{m} L A_{m}$ is the diagonal matrix:

$$
-\left(\begin{array}{ccc}
J_{1} & & 0 \\
& \ddots & \\
0 & & J_{p}
\end{array}\right),
$$

where $J_{k}=\lambda_{k}(t, x, \xi) I_{k}$ and $I_{k}$ is the $n_{k} \times n_{k}$ identity matrix, for $k=$ $1, \ldots, p$. In accordance with $(4.55)$, we write

$$
y=\left(\begin{array}{c}
y_{1} \\
\vdots \\
y_{p}
\end{array}\right),
$$

where $y_{k}$ is $\mathscr{C}^{n_{k}}$ valued, for $k=1, \ldots, p$. Now there is a conic neighborhood $\mathscr{O}_{m}$ of $\Gamma_{l}(t), t_{m} \leq t \leq t_{m+1}$, in $R^{n+1} \times\left(R^{n+1} \backslash\{0\}\right)$ such that

$$
\mathscr{O}_{m} \subset\left\{(t, x, \tau, \xi):(t, x, \xi) \in \mathscr{N}_{m}, \tau \in R\right\}
$$

and

$$
\tau^{2}-\lambda_{k}(t, x, \xi) \neq 0, \quad \text { for all }(t, x, \tau, \xi) \in \mathscr{O}_{m}, \text { if } k \neq l .
$$

We can also find a conic subset $\mathscr{O}_{m}^{\star}$ of $\mathscr{O}_{m}$ and $\gamma(t, x, \tau, \xi) \in \mathscr{S}^{0}\left(R^{n+1} \times R^{n+1}\right)$ such that

$$
\bigcup_{t_{m} \leq t \leq t_{m+1}} \Gamma_{l}(t) \subset \mathscr{O}_{m}^{*}
$$

(4.60) $\operatorname{supp} \gamma \subset \mathscr{O}_{m} \cap\left\{(t, x, \tau, \xi): \tau^{2}+|\xi|^{2} \geq \frac{1}{2},|\xi| \geq \varepsilon|\tau|\right.$, for some $\left.\varepsilon>0\right\}$,

$$
\gamma(t, x, \tau, \xi)=1, \quad \text { for }(t, x, \tau, \xi) \in \mathscr{O}_{m}^{\star} \text { satisfying } \tau^{2}+|\xi|^{2} \geq 1,
$$



$\gamma$ is homogeneous of degree zero in $(\tau, \xi)$ for $\tau^{2}+|\xi|^{2} \geq 1$.

By choosing a function $\psi(t) \in C_{0}^{\infty}((0, T))$ which satisfies

$$
\psi(t)=1 \text {, on an open interval containing }\left[t_{m}, t_{m+1}\right],
$$

we have from (4.43)

$$
\psi y_{t t}=\psi B_{m} L A_{m} y+\psi G_{1} y_{t}+\psi G_{2} y+\psi h \text { in } R^{n+1},
$$

and thus, by applying $\gamma\left(t, x, D_{t}, D_{x}\right)$ to both sides,

$$
Q_{k} y_{k}=\sum_{j=1}^{p} \Theta_{k, j} y_{j}+\sum_{j=1}^{p} \Xi_{k, j} y_{j t}+\beta_{k}, \quad \text { for } k=1, \ldots, p .
$$

Here $Q_{k}\left(t, x, D_{t}, D_{x}\right)$ is an $n_{k} \times n_{k}$ matrix operator in $O P S^{2}\left(R^{n+1}\right)$ such that

(4.66) the principal symbol of $Q_{k}=\gamma(t, x, \tau, \xi) \psi(t)\left(\tau^{2}-\lambda_{k}(t, x, \xi)\right) I_{k}$, for $\tau^{2}+|\xi|^{2} \geq c$, where $c$ is a positive constant and $I_{k}$ is the $n_{k} \times n_{k}$ identity matrix. $\boldsymbol{\Theta}_{k, j}$ and $\boldsymbol{\Xi}_{k, j}$ are $n_{k} \times n_{j}$ matrix operators such that

$$
\begin{aligned}
& \boldsymbol{\Theta}_{k, j} \in O P S^{1}\left(R^{n+1}\right), \\
& \Xi_{k, j} \in O P S^{0}\left(R^{n+1}\right),
\end{aligned}
$$

for $k, j=1, \ldots, p$. It follows from (4.54) that $\beta_{k}$ is a $\mathscr{C}^{n_{k}}$ valued function such that

$$
\beta_{k} \text { is } H^{\sigma-1} \text { at } \Gamma_{l}(t), \quad t_{m} \leq t \leq t_{m+1}, \quad \text { for } k=1, \ldots, p .
$$

For (4.67) through (4.69), we have used Lemma 4.4. By virtue of (4.52), (4.58), (4.61), (4.66) and (4.69), we can derive from (4.65)

$$
y_{k} \text { is } H^{\sigma} \text { at } \Gamma_{l}(t), t_{m} \leq t \leq t_{m+1},
$$

for $k \neq l$. Using this, we consider

$$
Q_{l} y_{l}-\Theta_{l, l} y_{l}-\Xi_{l, l} y_{l t}=\sum_{j \neq l} \Theta_{l, j} y_{j}+\sum_{j \neq l} \Xi_{l, j} y_{j t}+\beta_{l} \text {. }
$$

The right-hand side is $H^{\sigma-1}$ at $\Gamma_{l}(t), t_{m} \leq t \leq t_{m+1}$, on account of (4.69) and (4.70). Now we apply Proposition 3.5.1 of [8] to find that

$$
y_{l} \text { is } H^{\sigma} \text { at } \bigcup_{t_{m} \leq t \leq t_{m+1}} \Gamma_{l}(t) \text {. }
$$

Now (4.70) and (4.72) yield that

$$
w \text { is } H^{\sigma} \text { at } \bigcup_{t_{m} \leq t \leq t_{m+1}} \Gamma_{l}(t),
$$

and the proof is complete.

Remark 4.5. For Lemma 4.3, we cannot use Theorem 4.1 of $[15$, p. 135] unless all the distinct eigenvalues of $\left[C_{i j}^{\alpha \beta}(t, x) \xi_{\alpha} \xi_{\beta}\right]$ are simple. If there is a multiple eigenvalue, then at least one family of bicharacteristic curves of the scalar pseudodifferential operator $\operatorname{det}\left(\tau^{2} I-\left[C_{i j}^{\alpha \beta}(t, x) \xi_{\alpha} \xi_{\beta}\right]\right)$ degenerate into a single point. Such degenerate bicharacteristic curves are useless since our purpose is to relate the local regularity of solution near $t=0$ to the microlocal regularity of solution at a specified point.

Next we note a couple of technical facts. 
Lemma 4.6. Let $\mu \in R$. Suppose that $\psi(t) \in C_{0}^{\infty}((0, T)), \Psi\left(t, s, x, D_{x}\right) \in$ $O P S^{0}\left([0, T] \times[0, T] \times R^{n}\right)$ and $v \in C^{k}\left([0, T] ; H^{\mu-k}\left(R^{n}\right)\right)$, for all $k \in Z_{+}$. Then,

$$
\begin{gathered}
\psi v \in H^{\mu}\left(R^{n+1}\right), \\
\psi(t) \int_{0}^{t} \Psi\left(t, s, x, D_{x}\right) v(s) d s \in H^{\mu}\left(R^{n+1}\right) .
\end{gathered}
$$

Proof. If $\mu \leq 0$, then (4.74) and (4.75) follow directly from the Fourier transform. If $\mu$ is a positive integer, (4.74) and (4.75) are obvious. If $\mu$ is an arbitrary positive number, they follow by interpolation.

Lemma 4.7. Let $\mu \in R$ and $v \in C^{k}\left([0, T] ; H^{\mu-k}\left(R^{n}\right)\right)$, for every $k \in Z_{+}$. If $v$ is a solution of $(4.11)$ in $(0, T) \times R^{n}$, then

$$
\begin{aligned}
\left(\partial_{t}^{k} v\right)_{t t}= & L\left(\partial_{t}^{k} v\right)+\left(R_{1}+k L_{t} \widetilde{L}\right)\left(\partial_{t}^{k} v\right)_{t} \\
& +Q_{k, 0} \partial_{t}^{k} v+\cdots+Q_{k, k} v \\
& +\int_{0}^{t} \Phi_{k}(t, s) v(s) d s+f_{k},
\end{aligned}
$$

for each $k \in Z_{+}$, where $\widetilde{L}$ is a parametrix of $L$ as in (4.6) and

$$
\begin{gathered}
Q_{k, j}\left(t, x, D_{x}\right) \in O P S^{0}\left([0, T] \times R^{n}\right), \quad j=0,1, \ldots, k, \\
\Phi_{k}\left(t, s, x, D_{x}\right) \in O P S^{0}\left([0, T] \times[0, T] \times R^{n}\right), \\
f_{k} \in C^{\infty}\left([0, T] ; H^{m}\left(R^{n}\right)\right), \quad \text { for all } m \in Z_{+} .
\end{gathered}
$$

Proof. (4.76) can easily be shown by induction. We omit the details.

We proceed to prove the following result on the microlocal regularity along a bicharacteristic strip.

Lemma 4.8. Let $\mu \in R$ and suppose that $v$ is a solution of $(4.11)$ in $(0, T) \times R^{n}$ such that $v \in C^{k}\left([0, T] ; H^{\mu-k}\left(R^{n}\right)\right)$, for all $k \in Z_{+}$. Let $\Gamma_{l}(t)=(t, x(t), \tau(t)$, $\xi(t))$ be a bicharacteristic strip of $(0.1)$. If $(t, x(t))$ is disjoint from the singular support of $v$ at every $t \in[0, \varepsilon]$, for some $\varepsilon>0$, then

$$
\partial_{t}^{k} v \text { is } H^{\mu+1} \text { at } \Gamma_{l}(t), \quad \text { for each } 0<t<T,
$$

for all $k \in Z_{+}$.

Proof. We can partition the interval $[0, T]$ as above and apply Lemmas 4.3 and 4.6 to each subinterval successively to derive (4.80) for $k=0$. Now suppose that (4.80) is valid for $0 \leq k \leq N$, and set

$$
w=\partial_{t}^{N+1} v
$$

It follows from Lemmas 4.6 and 4.7 that

$$
w_{t t}=L w+\left(R_{1}+(N+1) L_{t} \widetilde{L}\right) w_{t}+Q_{N+1,0} w+y,
$$

where $y$ is $H^{\mu}$ at $\Gamma_{l}(t)$, for each $0<t<T$. Again by Lemma 4.3, we find that

$$
w \text { is } H^{\mu+1} \text { at } \Gamma_{l}(t), \quad 0<t<T .
$$

By induction, (4.80) is true for all $k \in Z_{+}$. 
4.3. Local regularity of $v$. Here we shall establish the following fact.

Proposition 4.9. Let $v$ be a solution of (4.11) in $(0, T) \times R^{n}$ such that for some $\mu \in R$,

$$
v \in C^{k}\left([0, T] ; H^{\mu-k}\left(R^{n}\right)\right),
$$

for all $k \in Z_{+}$. Let $\left(t^{*}, x^{*}\right) \in R^{n+1}, 0<t^{*}<T$, and assume that each bicharacteristic curve of $(0.1)$ passing through $\left(t^{*}, x^{*}\right)$ does not intersect the singular support of $v$ near $t=0$. Then, there is a function $\phi(t, x) \in C_{0}^{\infty}\left(R^{n+1}\right)$ which is identically 1 in a neighborhood of $\left(t^{*}, x^{*}\right)$ and such that

$$
\phi v \in C^{\infty}\left(R ; H^{\mu+2}\left(R^{n}\right)\right) .
$$

Proof. Choose any $\left(\tau^{*}, \xi^{*}\right) \in R^{n+1} \backslash\{0\}$. If $\mathscr{P}_{k}\left(t^{*}, x^{*}, \tau^{*}, \xi^{*}\right)=0$, for some $k=1, \ldots, p$, then there is a bicharacteristic strip passing through $\left(t^{*}, x^{*}, \tau^{*}\right.$, $\left.\xi^{*}\right)$ and the corresponding bicharacteristic curve does not intersect the singular support of $v$ near $t=0$. Therefore, we can apply Lemma 4.8 to find that

$$
\partial_{t}^{k} v \text { is } H^{\mu+1} \text { at }\left(t^{*}, x^{*}, \tau^{*}, \xi^{*}\right) \text {, }
$$

for all $k \in Z_{+}$. If $\mathscr{P}_{k}\left(t^{*}, x^{*}, \tau^{*}, \xi^{*}\right) \neq 0$, for each $k=1, \ldots, p$, then $D_{t}^{2}+L\left(t, x, D_{x}\right)$ is microlocally elliptic at $\left(t^{*}, x^{*}, \tau^{*}, \xi^{*}\right)$, and thus it follows from (4.11) that

$$
v \text { is } H^{\mu+2} \text { at }\left(t^{*}, x^{*}, \tau^{*}, \xi^{*}\right) .
$$

By induction, we can deduce from (4.76) that

$$
\partial_{t}^{k} v \text { is } H^{\mu+2} \text { at }\left(t^{*}, x^{*}, \tau^{*}, \xi^{*}\right),
$$

for every $k \in Z_{+}$. Combining (4.86) and (4.88), we have

$$
\partial_{t}^{k} v \text { is } H^{\mu+1} \text { at }\left(t^{*}, x^{*}\right) \text {, }
$$

for every $k \in Z_{+}$. Next, there is a positive number $\delta$ such that if

$$
\left|(\tilde{t}, \tilde{x})-\left(t^{*}, x^{*}\right)\right|<\delta,
$$

then any bicharacteristic curve passing through $(\tilde{t}, \tilde{x})$ does not meet the singular support of $v$ near $t=0$, and consequently,

$$
\partial_{t}^{k} v \text { is } H^{\mu+1} \text { at }(\tilde{t}, \tilde{x}),
$$

for every $k \in Z_{+}$. Hence, there is a positive number $\varepsilon$ such that

$$
v \in C^{\infty}\left(\left[t^{*}-\varepsilon, t^{*}+\varepsilon\right] ; H^{\mu+1}\left(B_{\varepsilon}\right)\right),
$$

where $B_{\varepsilon}=\left\{x \in R^{n}:\left|x-x^{*}\right|<\varepsilon\right\}$. Using the fact that $L\left(t, x, D_{x}\right)$ is strongly elliptic in $R^{n}$, we derive (4.85) from (4.11).

Proposition 4.10. In addition to the assumptions in Proposition 4.9, we further assume that

$$
x^{*} \notin \operatorname{sing} \operatorname{supp} v(0) \cup \operatorname{sing} \operatorname{supp} v_{t}(0) .
$$

Then, there is a function $\phi(t, x) \in C_{0}^{\infty}\left(R^{n+1}\right)$ which is identically 1 in a neighborhood of $\left(t^{*}, x^{*}\right)$ and such that

$$
\phi v \in C^{\infty}\left(R^{n+1}\right) .
$$

For the proof of this, we first observe the following fact. 
Lemma 4.11. Let $\mathscr{M}\left(t, s, x, D_{x}\right) \in O P S^{0}\left([0, T] \times[0, T] \times R^{n}\right)$. Under the same assumptions as in Proposition 4.10, we have

$$
\begin{aligned}
L(t)^{k} & \int_{0}^{t} \mathscr{M}(t, s) v(s) d s \\
& =\mathscr{M}_{k, 1}(t) v_{t}(t)+\mathscr{M}_{k, 2}(t) v(t)+\int_{0}^{t} \mathscr{H}_{k}(t, s) v(s) d s+\phi_{k},
\end{aligned}
$$

where the arguments $x$ and $D_{x}$ have been suppressed and

$$
\mathscr{M}_{k, 1}(t) \in \operatorname{OPS}^{2 k}\left([0, T] \times R^{n}\right),
$$

$$
\begin{gathered}
\mathscr{M}_{k, 2}(t) \in \operatorname{OPS}^{2 k}\left([0, T] \times R^{n}\right), \\
\mathscr{H}_{k}(t, s) \in O P S^{0}\left([0, T] \times[0, T] \times R^{n}\right), \\
\phi_{k} \in C^{\infty}\left([0, T] ; H^{\mu+1-2 k}\left(R^{n}\right)\right),
\end{gathered}
$$$$
\left\{\left(t, x^{*}\right): 0 \leq t \leq T\right\} \text { is disjoint from sing } \operatorname{supp} \phi_{k} .
$$

Proof. This is trivial for $k=0$. Suppose that (4.95) is valid for $0 \leq k \leq m$. Then,

$$
\begin{aligned}
L(t)^{m+1} & \int_{0}^{t} \mathscr{M}(t, s) v(s) d s \\
= & L(t) \mathscr{M}_{m, 1}(t) v_{t}(t)+L(t) \mathscr{M}_{m, 2}(t) v(t) \\
& +\int_{0}^{t} L(t) \mathscr{H}_{m}(t, s) \widetilde{L}(s) L(s) v(s) d s+\theta_{m},
\end{aligned}
$$

where $\widetilde{L}(s)$ is a parametrix of $L(s)$ and

$$
\theta_{m}=\int_{0}^{t} L(t) \mathscr{H}_{m}(t, s)(I-\widetilde{L}(s) L(s)) v(s) d s+L(t) \phi_{m} .
$$

By virtue of $(4.6),(4.84),(4.99)$ and $(4.100)$, it is easy to see that

$$
\theta_{m} \in C^{\infty}\left([0, T] ; H^{\mu-1-2 m}\left(R^{n}\right)\right),
$$

$$
\left\{\left(t, x^{*}\right): 0 \leq t \leq T\right\} \text { is disjoint from } \operatorname{sing} \operatorname{supp} \theta_{m} .
$$

Meanwhile, we can write

$$
\begin{aligned}
\int_{0}^{t} L(t) \mathscr{L}_{m}(t, s) \widetilde{L}(s) L(s) v(s) d s & \\
= & \int_{0}^{t} J(t, s)\left\{v_{s s}-R_{1}(s) v_{s}(s)-R_{2}(s) v(s)\right. \\
& \left.\quad+\int_{0}^{s} R_{s s}(s, \eta) v(\eta) d \eta-f(s)\right\} d s \\
= & J(t, t) v_{t}(t)-\left\{\left.J_{s}(t, s)\right|_{s=t}+J(t, t) R_{1}(t)\right\} v(t) \\
& -J(t, 0) v_{t}(0)+\left\{J_{s}(t, 0)+J(t, 0) R_{1}(0)\right\} v(0) \\
& +\int_{0}^{t} \mathscr{F}(t, s) v(s) d s-\int_{0}^{t} J(t, s) f(s) d s,
\end{aligned}
$$


where

(4.106)

$$
\begin{aligned}
J(t, s)= & L(t) \mathscr{H}_{m}(t, s) \tilde{L}(s) \in O P S^{0}\left([0, T] \times[0, T] \times R^{n}\right), \\
\mathscr{F}(t, s)= & J_{s s}(t, s)+\partial_{s}\left\{J(t, s) R_{1}(s)\right\} \\
& -J(t, s) R_{2}(s)+\int_{s}^{t} J(t, \eta) R_{\eta \eta}(\eta, s) d \eta \\
\in & O P S^{0}\left([0, T] \times[0, T] \times R^{n}\right) .
\end{aligned}
$$

Now we set

$$
\begin{aligned}
\phi_{m+1}= & -J(t, 0) v_{t}(0)+\left\{J_{s}(t, 0)+J(t, 0) R_{1}(0)\right\} v(0) \\
& -\int_{0}^{t} J(t, s) f(s) d s+\theta_{m} .
\end{aligned}
$$

Then, it follows from (4.15), (4.84), (4.93), (4.103) and (4.104) that

$$
\phi_{m+1} \in C^{\infty}\left([0, T] ; H^{\mu-1-2 m}\left(R^{n}\right)\right) \text {, }
$$

$$
\left\{\left(t, x^{*}\right): 0 \leq t \leq T\right\} \text { is disjoint from sing supp } \phi_{m+1} \text {. }
$$

We also set

$$
\begin{gathered}
\mathscr{M}_{m+1,1}(t)=L(t) \mathscr{M}_{m, 1}(t)+J(t, t) \\
\in O P S^{2 m+2}\left([0, T] \times R^{n}\right), \\
\mathscr{M}_{m+1,2}(t)=L(t) \mathscr{M}_{m, 2}(t)-\left.J_{s}(t, s)\right|_{s=t}-J(t, t) R_{1}(t) \\
\in O P S^{2 m+2}\left([0, T] \times R^{n}\right) .
\end{gathered}
$$

It is now apparent that the representation formula (4.95) is valid for $k=m+1$. By induction, it is valid for all $k \in Z_{+}$.

Proof of Proposition 4.10. We apply $L(t)^{k}$ to (4.11) and write, by Lemma 4.11,

$$
\begin{aligned}
L(t)^{k+1} v= & L(t)^{k} v_{t t}-L(t)^{k} R_{1}(t) v_{t}-L(t)^{k} R_{2}(t) v-L(t)^{k} f \\
& +R_{k, 1}(t) v_{t}+R_{k, 2}(t) v+\int_{0}^{t} \Phi_{k}(t, s) v(s) d s+\rho_{k},
\end{aligned}
$$

where

$$
R_{k, 1}(t) \in O P S^{2 k}\left([0, T] \times R^{n}\right)
$$$$
R_{k, 2}(t) \in O P S^{2 k}\left([0, T] \times R^{n}\right),
$$

Suppose that for some small positive numbers $\varepsilon$ and $\delta_{1}$,

$$
v \in C^{\infty}\left(\left[t^{*}-\varepsilon, t^{*}+\varepsilon\right] ; H^{\mu+2 m}\left(B_{\delta_{1}}\right)\right)
$$


where $B_{\delta_{1}}=\left\{x \in R^{n}:\left|x-x^{*}\right|<\delta_{1}\right\}$. Then, we use (4.84) and (4.113) through (4.118) with $k=m$ to deduce that

$$
v \in C^{\infty}\left(\left[t^{*}-\varepsilon, t^{*}+\varepsilon\right] ; H^{\mu+2 m+2}\left(B_{\delta_{2}}\right)\right),
$$

for some $0<\delta_{2}<\delta_{1}$. By induction, we can assert that $v$ is $C^{k}$ at $\left(t^{*}, x^{*}\right), \quad$ for each $k \in Z_{+}$.

As in (4.90), there is a positive number $\delta$ such that the same assumption as for $\left(t^{*}, x^{*}\right)$ holds for each $(\tilde{t}, \tilde{x})$ satisfying

$$
\left|(\tilde{t}, \tilde{x})-\left(t^{*}, x^{*}\right)\right|<\delta,
$$

and hence, for such $(\tilde{t}, \tilde{x})$,

$$
v \text { is } C^{k} \text { at }(\tilde{t}, \tilde{x}), \quad \text { for each } k \in Z_{+} .
$$

Now the proof of (4.94) is complete.

4.4. Conclusion of the proof of Theorem 1.5. We are now back to the statement of Theorem 1.5. Fix any $T>t^{*}$. According to Theorem 2.1, there is a unique solution $u(t, x)$ of $(0.1)$ and $(0.2)$ such that

$$
u \in C^{k}\left([0, T] ; H^{\nu-k}\left(R^{n}\right)\right),
$$

for every $k \in Z_{+}$. It follows from (4.9) that

$$
v \in C^{k}\left([0, T] ; H^{\nu-k}\left(R^{n}\right)\right),
$$

for every $k \in Z_{+}$. Now suppose that

$x_{0} \notin \operatorname{sing} \operatorname{supp} u_{0} \cup \operatorname{sing} \operatorname{supp} u_{1}$.

Then, by means of superposition of solutions, we can use Theorem 2.2 to find a positive number $\delta$ such that if $0 \leq t \leq \delta$ and $\left|x-x_{0}\right| \leq \delta$, then $(t, x) \notin$ sing supp $u$. Using the same $x_{0}$ and $\delta$, we can assert

Lemma 4.12. If $0 \leq \tilde{t}<\delta$ and $\left|\tilde{x}-x_{0}\right|<\delta$, then

$$
(\tilde{t}, \tilde{x}) \notin \operatorname{sing} \operatorname{supp} v \text {. }
$$

Proof. Fix such $(\tilde{t}, \tilde{x})$. Let $\varepsilon=\delta-\left|\tilde{x}-x_{0}\right|$, and choose $\psi_{1}(x)$ and $\psi_{2}(x)$ in $C_{0}^{\infty}\left(R^{n}\right)$ such that

$$
\begin{aligned}
& \psi_{1}(x)= \begin{cases}1, & \text { for }|x-\tilde{x}|<\varepsilon / 5, \\
0, & \text { for }|x-\tilde{x}|>\varepsilon / 4,\end{cases} \\
& \psi_{2}(x)= \begin{cases}1, & \text { for }|x-\tilde{x}|<\varepsilon / 3, \\
0, & \text { for }|x-\tilde{x}|>\varepsilon / 2\end{cases}
\end{aligned}
$$

Then, it follows from (4.9) that

$$
\begin{aligned}
\psi_{1} v= & \psi_{1} u+\int_{0}^{t} \psi_{1} Q\left(t, s, x, D_{x}\right)\left(1-\psi_{2}\right) u(s) d s \\
& +\int_{0}^{t} \psi_{1} Q\left(t, s, x, D_{x}\right) \psi_{2} u(s) d s .
\end{aligned}
$$

It is evident that $\psi_{1}(x) Q\left(t, s, x, D_{x}\right)\left(1-\psi_{2}(x)\right) \in O P S^{-\infty}\left([0, T] \times[0, T] \times R^{n}\right)$ and $\psi_{2}(x) u(t, x) \in C^{\infty}\left([0, \delta] ; H^{m}\left(R^{n}\right)\right)$, for all $m \in Z_{+}$, from which (4.127) follows. 
Hence, if a bicharacteristic curve does not intersect the singular support of $u_{0}$ and $u_{1}$ at $t=0$, then it does not intersect the singular support of $v$ near $t=0$. Now the hypotheses in Proposition 4.9 are satisfied, and

$$
\phi v \in C^{\infty}\left(R ; H^{\nu+2}\left(R^{n}\right)\right),
$$

for some $\phi \in C_{0}^{\infty}\left(R^{n+1}\right)$ which is identically 1 in a neighborhood of $\left(t^{*}, x^{*}\right)$. Next we write (4.10):

$$
u=v+\int_{0}^{t} R\left(t, s, x, D_{x}\right) v(s) d s .
$$

Combining (4.125), (4.131) and (4.132), we find that

$$
\phi u \in C^{\infty}\left(R ; H^{\nu}\left(R^{n}\right)\right) \text {. }
$$

We now improve the local regularity. It follows from (4.132) that

$$
\begin{aligned}
L(t) u= & L(t) v+\int_{0}^{t} L(t) R(t, s) \widetilde{L}(s) L(s) v(s) d s \\
& +\int_{0}^{t} L(t) R(t, s)(I-\widetilde{L}(s) L(s)) v(s) d s
\end{aligned}
$$

where the arguments $x$ and $D_{x}$ have been suppressed and $\widetilde{L}$ denotes a parametrix of $L$ as before. By virtue of (4.6) and (4.125), the last integral belongs to $C^{\infty}\left([0, T] ; H^{m}\left(R^{n}\right)\right)$ for every $m \in Z_{+}$. In the meantime, we use (4.105) to write

$$
\begin{aligned}
\int_{0}^{t} L(t) & R(t, s) \tilde{L}(s) L(s) v(s) d s \\
= & K(t, t) v_{t}(t)-\left\{\left.K_{s}(t, s)\right|_{s=t}+K(t, t) R_{1}(t)\right\} v(t) \\
& -K(t, 0) v_{t}(0)+\left\{K_{s}(t, 0)+K(t, 0) R_{1}(0)\right\} v(0) \\
& +\int_{0}^{t} \mathscr{G}(t, s) v(s) d s-\int_{0}^{t} K(t, s) f(s) d s
\end{aligned}
$$

where

$$
\begin{aligned}
K(t, s)= & L(t) R(t, s) \widetilde{L}(s) \in O P S^{0}\left([0, T] \times[0, T] \times R^{n}\right) \\
\mathscr{G}(t, s)= & K_{s s}(t, s)+\partial_{s}\left\{K(t, s) R_{1}(s)\right\} \\
& -K(t, s) R_{2}(s)+\int_{s}^{t} K(t, \eta) R_{\eta \eta}(\eta, s) d \eta \\
\in & O P S^{0}\left([0, T] \times[0, T] \times R^{n}\right)
\end{aligned}
$$

$$
f \in C^{\infty}\left([0, T] ; H^{m}\left(R^{n}\right)\right), \quad \text { for every } m \in Z_{+} .
$$

Meanwhile, it is apparent from (4.9) that

$$
v(0)=u_{0}, \quad v_{t}(0)=u_{1}+Q\left(0,0, x, D_{x}\right) u_{0},
$$

so that

$$
\left(v(0), v_{t}(0)\right) \in H^{\nu}\left(R^{n}\right) \times H^{\nu-1}\left(R^{n}\right) .
$$

We now combine (4.125), (4.131), (4.134) through (4.140) to obtain (1.24). 
Next we further assume that

$$
x^{*} \notin \operatorname{sing} \operatorname{supp} u_{0} \cup \operatorname{sing} \operatorname{supp} u_{1} .
$$

Then, by the pseudolocal property of $Q\left(0,0, x, D_{x}\right)$,

$$
x^{*} \notin \operatorname{sing} \operatorname{supp} v(0) \cup \operatorname{sing} \operatorname{supp} v_{t}(0) \text {. }
$$

Therefore, (4.94) holds. We then apply $L(t)^{k}$ to (4.10) and write, by Lemma 4.11,

$$
\begin{aligned}
L(t)^{k} u(t)= & L(t)^{k} v(t)+\Pi_{k, 1}(t) v_{t}(t)+\Pi_{k, 2}(t) v(t) \\
& +\int_{0}^{t} \mathscr{D}_{k}(t, s) v(s) d s+\varpi_{k}
\end{aligned}
$$

where

$$
\begin{gathered}
\Pi_{k, 1}(t) \in O P S^{2 k}\left([0, T] \times R^{n}\right), \\
\Pi_{k, 2}(t) \in O P S^{2 k}\left([0, T] \times R^{n}\right), \\
\mathscr{D}_{k}(t, s) \in O P S^{0}\left([0, T] \times[0, T] \times R^{n}\right), \\
\varpi_{k} \in C^{\infty}\left([0, T] ; H^{\nu+1-2 k}\left(R^{n}\right)\right), \\
\left\{\left(t, x^{*}\right): 0 \leq t \leq T\right\} \text { is disjoint from sing supp } \varpi_{k} .
\end{gathered}
$$

By means of (4.94), (4.125), and (4.143) through (4.148), we can argue as in the proof of Proposition 4.10 to arrive at (1.25).

Remark 4.11. We note that (4.135) indicates how the initial data can influence the local regularity of solutions through the vertical lines $\{(t, x): t \geq 0\}$ that intersect the singular support of the initial data.

4.5. Emergence of stationary singularities. In this subsection we will find a sufficient condition that ensures the emergence of stationary singularities. We choose arbitrarily large $T>0$, and rewrite (4.132) as

$$
\begin{aligned}
u= & +\int_{0}^{t} R(t, s) \widetilde{L}(s) L(s) v(s) d s \\
& +\int_{0}^{t} R(t, s)(I-\widetilde{L}(s) L(s)) v(s) d s,
\end{aligned}
$$

where the symbols have the same meaning as in (4.134), and the second integral belongs to $C^{\infty}\left([0, T] ; H^{m}\left(R^{n}\right)\right)$ for every $m \in Z_{+}$. As in (4.135), we can rewrite the first integral as

$$
\begin{aligned}
& \int_{0}^{t} R(t, s) \tilde{L}(s) L(s) v(s) d s \\
&= \mathscr{Y}(t, t) v_{t}(t)-\left\{\left.\mathscr{Y}_{s}(t, s)\right|_{s=t}+\mathscr{Y}(t, t) R_{1}(t)\right\} v(t) \\
&-\mathscr{Y}(t, 0) v_{t}(0)+\left\{\mathscr{Y}_{s}(t, 0)+\mathscr{Y}(t, 0) R_{1}(0)\right\} v(0) \\
&+\int_{0}^{t} \Upsilon(t, s) v(s) d s-\int_{0}^{t} \mathscr{Y}(t, s) f(s) d s,
\end{aligned}
$$


where

$$
\begin{aligned}
\mathscr{Y}(t, s) & =R(t, s) \widetilde{L}(s) \in O P S^{-2}\left([0, T] \times[0, T] \times R^{n}\right), \\
\Upsilon(t, s)= & \mathscr{Y}_{s s}(t, s)+\partial_{s}\left\{\mathscr{Y}(t, s) R_{1}(s)\right\} \\
& -\mathscr{Y}(t, s) R_{2}(s)+\int_{s}^{t} \mathscr{Y}(t, \eta) R_{\eta \eta}(\eta, s) d \eta \\
& \in O P S^{-2}\left([0, T] \times[0, T] \times R^{n}\right),
\end{aligned}
$$

$$
f \in C^{\infty}\left([0, T] ; H^{m}\left(R^{n}\right)\right), \quad \text { for every } m \in Z_{+},
$$

and $R_{1}$ and $R_{2}$ were defined by (4.12) and (4.13).

Now suppose that

$$
u_{0}=0, \quad u_{1} \in H^{\nu-1}\left(R^{n}\right) \backslash H^{\nu}\left(R^{n}\right) .
$$

Then, it follows from (4.139) that

$$
v(0)=0, \quad v_{t}(0) \in H^{\nu-1}\left(R^{n}\right) \backslash H^{\nu}\left(R^{n}\right),
$$

and hence there must be a point $x^{*}$ such that

$$
v_{t}(0) \text { is not } H^{\nu} \text { at } x^{*} \text {. }
$$

Let us suppose that there is $0<t^{*}<T$ such that any bicharacteristic curve passing through $\left(t^{*}, x^{*}\right)$ does not intersect the singular support of $v_{t}(0)$. By the same argument as for (4.131), it follows that

$$
v\left(t^{*}\right) \text { is } H^{\nu+2} \text { at } x^{*},
$$

and, by (4.151),

$$
\begin{gathered}
\mathscr{Y}\left(t^{*}, t^{*}\right) v_{t}\left(t^{*}\right) \text { is } H^{\nu+4} \text { at } x^{*}, \\
\left\{\left.\mathscr{Y}_{s}\left(t^{*}, s\right)\right|_{s=t^{*}}+\mathscr{Y}\left(t^{*}, t^{*}\right) R_{1}\left(t^{*}\right)\right\} v\left(t^{*}\right) \text { is } H^{\nu+4} \text { at } x^{*} .
\end{gathered}
$$

We also see that

$$
\int_{0}^{t^{*}} \Upsilon\left(t^{*}, s\right) v(s) d s \text { is } H^{\nu+2} \text { at } x^{*}
$$

We now assume that

$$
\left|\operatorname{det} R\left(t^{*}, 0, x^{*}, \xi\right)\right| \geq c_{1},
$$

for all $|\xi| \geq c_{2}$, for some $c_{1}, c_{2}>0$. Since $R\left(t^{*}, 0, x, D_{x}\right) \in O P S^{0}\left(R^{n}\right)$, (4.161) implies that $R\left(t^{*}, 0, x, D_{x}\right)$ is elliptic at $x^{*}$. Consequently,

$$
\mathscr{Y}\left(t^{*}, 0\right) v_{t}(0) \text { is } H^{\nu+1} \text { at } x^{*} \text {, but is not } H^{\nu+2} \text { at } x^{*} \text {. }
$$

Combining (4.157) through (4.162), we find from (4.149) that

$$
u\left(t^{*}\right) \text { is } H^{\nu+1} \text { at } x^{*} \text {, but is not } H^{\nu+2} \text { at } x^{*} \text {. }
$$

The singularity described by (4.163) is a stationary singularity, because any bicharacteristic curve passing through $\left(t^{*}, x^{*}\right)$ does not intersect the singular support of the initial data and because the location of singularity of the initial data at $x^{*}$ has not changed as time evolved. If there is $c>0$ such that the above assumptions on $t^{*}$ are satisfied for each $t^{*} \geq c$ with the fixed $x^{*}$, then (4.162) is true for all $t^{*} \geq c$ with the fixed $x^{*}$. This can be easily implemented if $v_{t}(0)$ has compact support and (4.161) holds for all large $t^{*}$. Hence ve have proved the following. 
Proposition 4.12. Suppose that the initial data have compact support, and satisfy (4.154) and (4.156). If (4.161) holds for all large $t^{*}$, where the constants $c_{1}$ and $c_{2}$ may depend on $t^{*}$, then a stationary singularity exists and persists permanently.

\section{REFERENCES}

1. R. Beals, Characterization of pseudodifferential operators and applications, Duke Math. J. 44 (1977), 45-57; correction 46 (1979), p. 215.

2. B. D. Coleman, M. E. Gurtin, and I. R. Herrera, Waves in materials with memory, Arch. Rational Mech. Anal. 19 (1965), 1-19, 239-265.

3. C. M. Dafermos, An abstract Volterra equation with applications to linear viscoelasticity, J. Differential Equations 7 (1970), 554-569.

4. G. F. D. Duff, The Cauchy problem for elastic waves in an anisotropic medium, Philos. Trans. Roy. Soc. London Ser. A 252 (1960), 249-273.

5. J. M. Greenberg, L. Hsiao and R. C. MacCamy, A model Riemann problem for Volterra equations, Volterra and Functional Differential Equations (K. Hannsgen et al., eds.), Marcel Dekker, New York, 1982, pp. 25-43.

6. G. Gripenberg, S-O. Londen, and O. Staffans, Volterra integral and functional equations, Cambridge Univ. Press, Cambridge, 1990.

7. K. B. Hannsgen and R. L. Wheeler, Behavior of the solutions of a Volterra equation as a parameter tends to infinity, J. Integral Equations 7 (1984), 229-237.

8. L. Hörmander, On the existence and the regularity of solutions of linear pseudo-differential equations, Enseign. Math. (2) 17 (1971), 99-163.

9. __ The analysis of linear partial differential operators. Vol. 3, Springer-Verlag, Berlin, 1985.

10. W. J. Hrusa and M. Renardy, On wave propagation in linear viscoelasticity, Quart. Appl. Math. 43 (1985), 237-254.

11. J. U. Kim, Local regularity of the one-dimensional motion of a viscoelastic medium, SIAM J. Math. Anal. (to appear).

12. R. C. MacCamy, A model Riemann problem for Volterra equations, Arch. Rational Mech. Anal. 82 (1983), 71-86.

13. M. Renardy, Some remarks on the propagation and non-propagation of discontinuities in linearly viscoelastic liquids, Rheology Acta 21 (1982), 251-254.

14. M. Renardy, W. J. Hrusa, and J. A. Nohel, Mathematical problems in viscoelasticity, Longman, New York, 1986.

15. M. E. Taylor, Pseudodifferential operators, Princeton Univ. Press, Princeton, NJ, 1981.

16. __ Rayleigh waves in linear elasticity as a propagation of singularities phenomenon, Partial Differential Equations and Geometry (Proc. Conf., Park City, UT, 1977; C. I. Byrnes, ed.), Marcel Dekker, New York, 1979, pp. 273-291.

Department of Mathematics, Virginia Polytechnic Institute and State University, BLACKSBURG, ViRgINIA 24061-0123

E-mail address: kim@math.vt.edu 